\title{
Taming hyperchaos with ESDDFD discretization of a conformable fractional derivative financial system with market confidence and ethics risk
}

\author{
D.P. Clemence-Mkhope and Gregory A. Gibson \\ Department of Mathematics and Statistics, North Carolina A\&T State University, Greensboro, \\ NC 27411. E-mail: clemence@ncat.edu, ggibson@ncat.edu
}

\begin{abstract}
Four discrete models using the exact spectral derivative discretization finite difference (ESDDFD) method are proposed for a chaotic five-dimensional, conformable fractional derivative financial system incorporating ethics and market confidence. Since the system considered was recently studied using the conformable Euler finite difference (CEFD) method and found to be hyperchaotic, and the CEFD method was recently shown to be valid only at fractional index $\alpha=1$, the source of the hyperchaos is in question. Through numerical experiments, illustration is presented that the hyperchaos previously detected is in part an artifact of the CEFD method as it is absent from the ESDDFD models.
\end{abstract}

Keywords: Conformable calculus; Fractional-order financial system; ESDDFD and NSFD methods; Hyperchaotic attractor; Market confidence; Ethics risk

\section{Introduction}

Hyperchaotic systems $[19,20]$, typically defined as systems with at least two positive Lyapunov exponents [1-3], of fractional-order have been investigated in many contexts, such as systems of Rössler [4] or Lorenz [9] type, those with flux controlled memristors [5] or realized in circuits [6-8], those arising from cellular neural networks [10], and financial systems [Xin et al., 2019]. As recounted in [Xin et al., 2019], nonlinear financial system depicting the relationship among interest rates, investments, prices, and savings was first introduced by Huang and Li [11]. It was extended to fractional order in Chen [12], to uncertain fractional-order form in Wang, Huang and Shen [13], to delayed form in Mircea et al. [14], and discrete form in Xin, Chen, and Ma [15]. Average profit margin was added as a variable in Yu, Cai, and $\mathrm{Li}[16]$ while investment incentive and market confidence were introduced in Xin, Li, and Zhang [17, 18]. Xin and Zhang [18] updated the 3-dimensional Huang and Li [11] model to a 4-dimensional one by accounting for market confidence, and [Xin et al., 2019] incorporated ethics risk to obtain a 5dimensional system, which was then fractionalized to obtain the following fractional-order hyperchaotic financial system considered in [Xin et al., 2019]:

$T_{t}^{\alpha_{1}} x=z+(y-a) x+k(w-p u)$

$T_{t}^{\alpha_{2}} y=1-b y-x^{2}+k(w-p u)$

$T_{t}^{\alpha_{3}} z=-x-c z+k(w-p u)$

$T_{t}^{\alpha_{4}} w=-d x y z$

$T_{t}^{\alpha_{5}} u=k(w-p u)$

where $\alpha=(\alpha 1, \alpha 2, \alpha 3, \alpha 4, \alpha 5)$ is subject to $\alpha 1, \alpha 2, \alpha 3, \alpha 4, \alpha 5 \in(0,1)$, and $T_{t}^{\alpha_{i}}, 1 \leq i \leq 5$, denotes the conformable fractional derivative of order $\alpha_{i}$. The variables $\mathrm{x}, \mathrm{y}, \mathrm{z}, \mathrm{w}, \mathrm{u}$ are the interest rate, investment 
demand, price index, market confidence, and ethics risk; the parameters $a, b, c$ are the saving amount, cost per investment, and demand elasticity of commercial markets, respectively, and a, b, c $\geq 0 ; k, p, d$ are impact factors associated with ethics risk.

Once proposed, and since analytic solutions do not exists, suitable numerical schemes to obtain solutions of the conformable derivative financial system. Though there are several methods to solve a conformable derivative system [41,51-72], these are too complex for many people. Inspired by the discretization process for the Caputo derivative for Ricatti equations [73] and Chua systems [74], the conformable Euler's finite difference (CEFD) method [75] for the five-dimensional fractional-order financial system is proposed in [1]. Numerical experiments with the resulting discrete model were conducted to detect a hyperchaotic attractor of the system. However, the standard Euler discretization of integer order systems such as studied in [1] is known to induce (see, e.g., [Garba et al], Mick, 2020]) numerical instabilities and spurious behavior where none exists in the continuous system. Moreover, the CEFD method has recently shown [DPC-M \& BGBC-M] to be valid only for $\alpha=1$ and is therefore not a valid fractional method. Nonstandard finite difference (NSFD) models have extensively [Mick 2020] been shown to eliminate induced chaos; the ESDDFD methodology is a novel extension, developed in the context of advection-reaction-diffusion equations [DPC-M, 2021 arXiv(a)], of the NSFD method to non-integer derivatives [DPC-M, $2021 \operatorname{arXiv(b)].~}$

It is therefore natural to ask whether some of the hyperchaotic behavior detected in the fractional financial system is an artifact of the method, and whether ESDDFD models can be constructed to eliminate such induced hyperchaos. The purpose of the present study is to investigate this question, in particular the effects of the discretization of the derivative and that of non-linear terms. To this end, the following four discrete models using the ESDDFD method are constructed for the system (1.1) and the experiments of [Xin et al., 2019] are repeated with the new models.

$$
\begin{aligned}
& \frac{x_{k+1}-x_{k}}{\phi_{j}\left(h, \alpha_{1}\right)}=F_{i}^{x}\left(x_{k}, y_{k}, z_{k}, u_{k}, w_{k}\right) \\
& \frac{y_{k+1}-y_{k}}{\phi_{j}\left(h, \alpha_{2}\right)}=F_{i}^{y}\left(x_{k}, y_{k}, z_{k}, u_{k}, w_{k}\right), \\
& \frac{z_{k+1}-z_{k}}{\phi_{j}\left(h, \alpha_{3}\right)}=-x_{k}-c z_{k}+k\left(w_{k}-p u_{k}\right), \\
& \frac{u_{k+1}-u_{k}}{\phi_{j}\left(h, \alpha_{5}\right)}=k\left(w_{k}-p u_{k}\right) \\
& \frac{w_{k+1}-w_{k}}{\phi_{j}\left(h, \alpha_{4}\right)}=F_{i}^{w}\left(x_{k}, y_{k}, z_{k}, z_{k}\right) \\
& i=1,2 \text { and } j=1,2, \text { where, } \\
& F_{1}^{x}\left(x_{k}, y_{k}, z_{k}, u_{k}, w_{k}\right)=z_{k}+\left(y_{k+1}-\mathrm{a}\right) x_{k}+k\left(w_{k}-p u_{k}\right) \\
& F_{1}^{y}\left(x_{k}, y_{k}, z_{k}, u_{k}, w_{k}\right)=1-b y_{k}-x_{k} x_{k}+k\left(w_{k}-p u_{k}\right), F_{1}^{w}\left(x_{k}, y_{k}, z_{k}, z_{k}\right)=-\frac{d}{2} x_{k} y_{k}\left(z_{k}+z_{k}\right) \\
& F_{2}^{x}=F_{1}^{x}\left(x_{k}, y_{k+1}, z_{k}, u_{k}, w_{k}\right), F_{2}^{y}=F_{1}^{y}\left(x_{k}, y_{k+1}, z_{k}, u_{k}, w_{k}\right), F_{2}^{w}=F_{1}^{w}\left(x_{k}, y_{k+1}, z_{k}, z_{k+1}\right)
\end{aligned}
$$

The remainder of this article is organized as follows. In Sect. 2, ESDDFD fundamentals, description of the model (1.1), and the CEFD model from [1] are presented. Section 3 presents construction of the denominator functions, $\phi_{j}\left(h, \alpha_{m}\right), 1 \leq m \leq 5$, for the ESDDFD model (1.2) and compares sub-models of (1.2) with corresponding CEFD sub-models. In Sect. 4, experimental results are 
presented of hyperchaotic attractor detection from the proposed financial system using both methods. Some concluding remarks in Sect. 5 close the paper.

\section{Preliminaries}

\subsection{The conformable derivative ESDDFD discrete model construction fundamentals}

While the Riemann-Liouville, Caputo, Atangana-Belaneau, and Grunwald-Letnikov fractional derivatives [21-28] are widely used in various applications, their definitions lack the chain rule, a classical derivative property satisfied by the conformable fractional derivative (CFD) [29-31] and its various extensions (see, e.g., [ Imb, 2019]). A financial system with market confidence and ethics risk model was recently [1] added to the many existing applications of the CFD in various scientific fields [32-42].

\subsection{The conformable derivative hyperchaotic financial system and its CEFD model}

The conformable fractional derivative financial system model (1.1) is based on a successive addition of various factors starting with the Huang and Li [11] nonlinear financial system model:

$x=z+(y-a) x$

$y^{\prime}=1-b y-x 2$,

$z^{*}=-x-c z$

modeling the interaction of interest rate, investment demand, and price index; the variables and parameters are the same as in (1.1). Model (2.1) was extended by Xin and Zhang [18] to account for market confidence:

$x=z+(y-a) x+m 1 w$,

$y^{\prime}=1-b y-x 2+m 2 w$,

$z^{\prime}=-x-c z+m 3 w$,

$w^{\cdot}=-x y z$,

where $\mathrm{m} 1, \mathrm{~m} 2, \mathrm{~m} 3$ are the impact factors associated with market confidence; the remaining variables and parameters are the same as in (2.1). Model (1.1) is the fractionalization, predicated on the practice that fractional-order economic systems [18, 76-80] can generalize their integer-order forms [17, 81, 82], of the following extension of (2.2) in [1] to account for both market confidence and ethics risk:

$x=z+(y-a) x+k(w-p u)$,

$y^{\cdot}=1-b y-x 2+k(w-p u)$,

$z^{\prime}=-x-c z+k(w-p u)$,

$u^{\cdot}=k(w-p u)$.

$w^{*}=-d x y z$,

When $\alpha=(1,1,1,1,1)$, system (1.1) degenerates to system (2.3); in the absence of ethics risk, (2.3) reduces to (2.2); in the absence of market confidence, (2.2) reduces to (2.1). In these three cases, 
therefore, any discrete method developed for (1.1) must reduce to that of the respective three reduced systems. Chaotic behavior for both the CEFD and ESDDFD models will be investigated in Section 3 for (1.1) as well as the three reduced systems (2.1)-(2.3).

The following discrete model was obtained in [1] from the CEFD method and used to investigate hyperchaos of the system (1.1):

$$
\begin{aligned}
& x_{k+1}=x_{k}+\frac{h^{\alpha_{1}}}{\alpha_{1}}\left(z_{k}+\left(y_{k}-a\right) x_{k}+k\left(w_{k}-p u_{k}\right)\right) \\
& y_{k+1}=y_{k}+\frac{h^{\alpha_{2}}}{\alpha_{2}}\left(1-b y_{k}-x_{k} x_{k}+k\left(w_{k}-p u_{k}\right)\right) \\
& z_{k+1}=z_{k}-\frac{h^{\alpha_{3}}}{\alpha_{3}}\left(x_{k}+c z_{k}-k\left(w_{k}-p u_{k}\right)\right) \\
& u_{k+1}=u_{k}+\frac{h^{\alpha_{5}}}{\alpha_{5}} k\left(w_{k}-p u_{k}\right) \\
& w_{k+1}=w_{k}-\frac{h^{\alpha_{4}}}{\alpha_{4}} d x_{k} y_{k} z_{k}
\end{aligned}
$$

\section{ESDDFD Discretization of conformable derivative system and its reductions}

In the ESDDFD and NSFD discretization methodologies, the first step is to consider a linear sub-system whose exact or best scheme can be constructed. Such a sub-system in this case is the following,

$T_{t}^{\alpha_{1}} x=-a x$

$$
\begin{array}{ll}
T_{t}^{\alpha_{2}} y=-b y, & T_{t}^{\alpha_{3}} z=-c z, \\
T_{t}^{\alpha_{5}} u=-k p u, &
\end{array}
$$

$T_{t}^{\alpha_{4}} w=0$,

which has only positive solutions for any positive initial data. The exact discretization of (3.1), which has a solution identical to that of (3.1), is as follows:

$$
\begin{array}{lll}
\frac{x_{k+1}-x_{k}}{\phi_{1}\left(h, \alpha_{1}\right)}=-a x_{k}, & \frac{y_{k+1}-y_{k}}{\phi_{1}\left(h, \alpha_{2}\right)}=-b y_{k}, & \frac{z_{k+1}-z_{k}}{\phi_{1}\left(h, \alpha_{3}\right)}=-c z_{k}, \\
\frac{w_{k+1}-w_{k}}{\phi_{1}\left(h, \alpha_{4}\right)}=0, & \frac{u_{k+1}-u_{k}}{\phi_{1}\left(h, \alpha_{5}\right)}=-k p u_{k},
\end{array}
$$

where the nonstandard denominators $\phi_{1}\left(h, \alpha_{i}\right), 1 \leq i \leq 5$, are given by

$\phi_{1}\left(h, \alpha_{i}\right)=\frac{1}{Q_{i}}\left(1-e^{-\frac{Q_{i}}{\alpha_{i}}\left[(t+h)^{\alpha_{i}} t^{\left.\alpha_{i}\right]}\right.}\right)$, with $Q_{1}=a, Q_{2}=b, Q_{3}=c, Q_{4}=0, Q_{5}=k p$

Since (1.1) reduces to (3.1), any valid discrete model for (1.1) must be reducible to one consistent with its exact discretization, that is, (3.2). By comparison, a reduction of the CEFD model (2.4) to the sub-system (3.1) yields the following discrete sub-system:

$$
\begin{array}{ll}
x_{k+1}=x_{k}-\frac{h^{\alpha_{1}}}{\alpha_{1}} a x_{k}, & y_{k+1}=y_{k}-\frac{h^{\alpha_{2}}}{\alpha_{2}} b y_{k}, \\
w_{k+1}=w_{k}+Q_{4} \frac{h^{\alpha_{4}}}{\alpha_{4}} w_{k}, & u_{k+1}=u_{k}-\frac{h^{\alpha_{5}}}{\alpha_{5}} k p u_{k},
\end{array}
$$


which is positive only if the following condition is satisfied: $\left(1-\frac{h^{\alpha_{i}}}{\alpha_{i}} Q_{i}\right) \geq 0,1 \leq i \leq 5$, with the $Q_{i}$ as in (3.2); such conditional positivity is known to induce chaotic behavior. All the sub-equations. (3.2) are of the form

$T_{t}^{\alpha} P=-\lambda P$,

whose CEFD scheme is

$P_{k+1}=P_{k}-\frac{h^{\alpha}}{\alpha} \lambda P_{k}$

which has been conclusively shown in [DPC-M \& BGBC-M] to be valid only for $\alpha=1$.

It is shown in [DPC-M \& BGBC-M] that a modified CEFD (MCEFD) may be obtained from the following alternate CFD definition, which is equivalent to the fractional change of variables in the integer-valued derivative (see also [Ulnes et al]):

Definition 1. Given a real-valued function on $[0, \infty)$, the conformable fractional derivative has the following alternative definition:

${ }_{0}^{C} T_{t}^{\alpha}[f(t)] \equiv \lim _{h \rightarrow 0}{ }^{C F D} \Delta_{t}^{\alpha}[f(t)]=\alpha \lim _{h \rightarrow 0} \frac{f(t+h)-f(t)}{\left[(t+h)^{\alpha}-t^{\alpha}\right]}$,

where ${ }_{0}^{C} T_{t}^{\alpha}[f(0)]$ is understood to mean ${ }_{0}^{C} T_{t}^{\alpha}[f(0)]=\lim _{t \rightarrow 0^{+}}{ }_{0}^{C} T_{t}^{\alpha}[f(t)]$.

The Euler scheme resulting from the MCFED is therefore the same as that given in Eqn. (??), only with the denominators

$$
\phi_{1}\left(h, \alpha_{i}\right)=\frac{1}{Q_{i}}\left(1-e^{-\frac{Q_{i}}{\alpha_{i}}\left[(t+h)^{\alpha_{i}-t^{\left.\alpha_{i}\right]}}\right.}\right)
$$

replaced by

$$
\phi_{2}\left(h, \alpha_{i}\right)=\frac{1}{\alpha_{i}}\left[(t+h)^{\alpha_{i}}-t^{\alpha_{i}}\right], 1 \leq i \leq 5,
$$

which is equivalent to replacing $h^{\alpha_{i}}$ by $\alpha_{i} \phi_{2}\left(h, \alpha_{i}\right)$ in the CEFD scheme (3.??).

To enable assessment of the effect of the denominators $\phi_{j}\left(h, \alpha_{i}\right), j=1,2$, the following schemes are compared:

$\frac{x_{k+1}-x_{k}}{\phi_{j}\left(h, \alpha_{1}\right)}=z_{k}+\left(y_{k}-\mathrm{a}\right) x_{k}$,

$\frac{y_{k+1}-y_{k}}{\phi_{j}\left(h, \alpha_{2}\right)}=1-b y_{k}-\left(x_{k}\right)^{2}$,

$\frac{z_{k+1}-z_{k}}{\phi_{j}\left(h, \alpha_{3}\right)}=-x_{k}-c z_{k}, j=1,2$.

To enable assessment of the effect of the non-local discretization of nonlinear terms, the following schemes are compared:

$\frac{x_{k+1}-x_{k}}{\phi_{j}\left(h, \alpha_{1}\right)}=z_{k}+\left(y_{k+1}-\mathrm{a}\right) x_{k}$

$\frac{y_{k+1}-y_{k}}{\phi_{j}\left(h, \alpha_{2}\right)}=1-b y_{k}-x_{k+1} x_{k}$

$\frac{z_{k+1}-z_{k}}{\phi_{j}\left(h, \alpha_{3}\right)}=-x_{k}-c z_{k}, j=1,2$. 
The terms $(y-\mathrm{a}) x$, and $\mathrm{x}^{2}$ are discretized non-locally as, respectively, $\left(y_{k+1}-\mathrm{a}\right) x_{k}$ and $x_{k+1} x_{k}$, while discretization of the terms $z$ in Eqns. (3.4a) and $x$ in Eqn. (3.4c) as $z_{k}$ and $x_{k}$ ensures respective consistency with the terms $c z$ of in Eqn. (3.4c) and $a x$ in Eqn. (3.4a in the cases $c=1$ and $a=1$.

By comparison, the scheme obtained by a reduction of the CEFD model (2.4) to its 3-dimensional sub-system (2.1) yields the following discrete sub-system:

$$
\begin{aligned}
& x_{k+1}=x_{k}+\frac{h^{\alpha_{1}}}{\alpha_{1}}\left(z_{k}+\left(y_{k}-\mathrm{a}\right) x_{k}\right) \\
& y_{k+1}=y_{k}+\frac{h^{\alpha_{2}}}{\alpha_{2}}\left(1-b y_{k}-x_{k} x_{k}\right) \\
& z_{k+1}=z_{k}+\frac{h^{\alpha_{3}}}{\alpha_{3}}\left(-x_{k}-c z_{k}\right) .
\end{aligned}
$$

Since system (3.5) reduces to the $x-y-z$ sub-system of (3.3), which suffers from induced chaos, it is to be expected that it too suffers the same, which will be numerically investigated in the next section.

The ESDDFD models (1.2) are then obtained by discretizing $k(w-p u)$ as $k\left(w_{k}-p u_{k}\right)$ to ensure consistency with (3.2) and then discretizing $x y z$ non-locally as either $\frac{1}{2} x_{k} y_{k}\left(z_{k}+z_{k}\right)$ or $\frac{1}{2} x_{k} y_{k+1}\left(z_{k}+z_{k+1}\right)$, where the form $x_{k} y_{k+1}$ is used to match the $x y$ term in the $x$-equation.

$$
\begin{aligned}
& \frac{x_{k+1}-x_{k}}{\phi_{j}\left(h, \alpha_{1}\right)}=z_{k}+\left(y_{k}-\mathrm{a}\right) x_{k}+k\left(w_{k}-p u_{k}\right) \\
& \frac{y_{k+1}-y_{k}}{\phi_{j}\left(h, \alpha_{2}\right)}=1-b y_{k}-\left(x_{k}\right)^{2}+k\left(w_{k}-p u_{k}\right) \\
& \frac{z_{k+1}-z_{k}}{\phi_{j}\left(h, \alpha_{3}\right)}=-x_{k}-c z_{k}+k\left(w_{k}-p u_{k}\right) . \\
& \frac{u_{k+1}-u_{k}}{\phi_{j}\left(h, \alpha_{5}\right)}=k\left(w_{k}-p u_{k}\right), \\
& \frac{w_{k+1}-w_{k}}{\phi_{j}\left(h, \alpha_{4}\right)}=-\frac{d}{2} x_{k} y_{k}\left(z_{k}+z_{k}\right), j=1,2 . \\
& \text { and } \\
& \frac{x_{k+1}-x_{k}}{\phi_{j}\left(h, \alpha_{1}\right)}=z_{k}+\left(y_{k+1}-\mathrm{a}\right) x_{k}+k\left(w_{k}-p u_{k}\right) \\
& \frac{y_{k+1}-y_{k}}{\phi_{j}\left(h, \alpha_{2}\right)}=1-b y_{k}-x_{k+1} x_{k}+k\left(w_{k}-p u_{k}\right) \\
& \frac{z_{k+1}-z_{k}}{\phi_{j}\left(h, \alpha_{3}\right)}=-x_{k}-c z_{k}+k\left(w_{k}-p u_{k}\right) . \\
& \frac{u_{k+1}-u_{k}}{\phi_{j}\left(h, \alpha_{5}\right)}=k\left(w_{k}-p u_{k}\right), \\
& \frac{w_{k+1}-w_{k}}{\phi_{j}\left(h, \alpha_{4}\right)}=-\frac{d}{2} x_{k} y_{k+1}\left(z_{k}+z_{k+1}\right), j=1,2 .
\end{aligned}
$$

The schemes (????) are explicit and can be explicitly solved for each $j=1,2$, in the order $x_{k+1}, y_{k+1}, z_{k+1}, u_{k+1}, w_{k+1}$ to obtain the following:

$$
x_{k+1}=x_{k}+\phi_{j}\left(h, \alpha_{1}\right)\left[z_{k}+\left(y_{k}-\mathrm{a}\right) x_{k}+k\left(w_{k}-p u_{k}\right)\right]
$$




$$
\begin{aligned}
& y_{k+1}=y_{k}+\phi_{j}\left(h, \alpha_{2}\right)\left[1-b y_{k}-\left(x_{k}\right)^{2}+k\left(w_{k}-p u_{k}\right)\right] \\
& z_{k+1}=z_{k}-\phi_{j}\left(h, \alpha_{3}\right)\left[x_{k}+c z_{k}-k\left(w_{k}-p u_{k}\right)\right] . \\
& u_{k+1}=u_{k}+\phi_{j}\left(h, \alpha_{5}\right)\left[k\left(w_{k}-p u_{k}\right)\right], \\
& w_{k+1}=w_{k}-\frac{d}{2} \phi_{j}\left(h, \alpha_{4}\right) x_{k} y_{k}\left(z_{k}+z_{k}\right), j=1,2 .
\end{aligned}
$$

While implicit, the schemes (????) can be explicitly solved for each $j=1,2$ in the order $u_{k+1}, z_{k+1}, x_{k+1}, y_{k+1}, w_{k+1}$ to obtain the following:

$u_{k+1}=u_{k}+\phi_{j}\left(h, \alpha_{5}\right)\left[k\left(w_{k}-p u_{k}\right)\right]$

$z_{k+1}=z_{k}-\phi_{j}\left(h, \alpha_{3}\right)\left[x_{k}+c z_{k}-k\left(w_{k}-p u_{k}\right)\right]$

$x_{k+1}=\frac{1}{\left[1+\phi_{j}\left(h, \alpha_{1}\right) x_{k} \phi_{j}\left(h, \alpha_{2}\right) x_{k}\right]}\left(x_{k}+\phi_{j}\left(h, \alpha_{1}\right) x_{k}\left\{y_{k}+\phi_{j}\left(h, \alpha_{2}\right)\left[1-b y_{k}+k\left(w_{k}-p u_{k}\right)\right]\right\}\right)$

$+\frac{1}{\left[1+\phi\left(h, \alpha_{1}\right) x_{k} \phi\left(h, \alpha_{2}\right) x_{k}\right]} \phi_{j}\left(h, \alpha_{1}\right)\left[z_{k}-\mathrm{a} x_{k}+k\left(w_{k}-p u_{k}\right)\right]$

$w_{k+1}=w_{k}-\phi_{j}\left(h, \alpha_{4}\right) \frac{d}{2} x_{k} y_{k+1}\left(z_{k}+z_{k+1}\right)$

\section{Numerical Experiments}

In this section, hyperchaos detection experiments are conducted parallel to those of [1] by varying the parameters related to ethics risk, such as $\alpha 5$, the confidence factor $k$, and the risk factor $p$, in the CEFD and ESDDFD models and their reductions. The following parameters and initial point values are fixed following [1]: $h=0.002, a=0.8, b=0.6, c=1, d=2, \alpha 1=0.3, \alpha 2=0.5, \alpha 3=0.6, \alpha 4=0.24, x 0=0.4, y 0=$ $0.6, \mathrm{z} 0=0.8, \mathrm{w} 0=0.3, \mathrm{u} 0=0.4$.

\subsection{Three-dimensional systems comparison}

There were no experiments performed in [Xin et al., 2019] for this case [Xin et al., 2019]. Simulations for both the ESDDFD model (3.4) and CEFD model (3.5) are performed with the same parameters. The following models $(4.1)-(4.4)$, obtained by the ESDDFD method,

$$
\begin{aligned}
& \frac{x_{k+1}-x_{k}}{\frac{1}{0.8}\left[1-e^{\frac{-0.8}{0.3}\left[(t+h)^{0.3}-t^{0.3}\right]}\right]}=z_{k}+\left(y_{k}-0.8\right) x_{k}, \\
& \frac{y_{k+1}-y_{k}}{\frac{1}{0.6}\left[1-e^{\frac{-0.6}{0.5}\left[(t+h)^{0.5}-t^{0.5}\right]}\right]}=1-0.6 y_{k}-\left(x_{k}\right)^{2}, \\
& \frac{z_{k+1}-z_{k}}{\left[1-e^{\frac{-1}{0.6}\left[(t+h)^{0.6}-t^{0.6}\right]}\right]}=-x_{k}-z_{k} \\
& \frac{x_{k+1}-x_{k}}{\frac{1}{0.3}\left[(t+h)^{0.3}-t^{0.3}\right]}=z_{k}+\left(y_{k}-0.8\right) x_{k},
\end{aligned}
$$




$$
\begin{aligned}
& \frac{y_{k+1}-y_{k}}{\frac{1}{0.5}\left[(t+h)^{0.5}-t^{0.5}\right]}=1-0.6 y_{k}-\left(x_{k}\right)^{2} \text {, } \\
& \frac{z_{k+1}-z_{k}}{\frac{1}{0.6}\left[(t+h)^{0.6}-t^{0.6}\right]}=-x_{k}-z_{k} \\
& \frac{x_{k+1}-x_{k}}{\frac{1}{0.8}\left[1-e^{\frac{-0.8}{0.3}\left[(t+h)^{0.3}-t^{0.3}\right]}\right]}=z_{k}+\left(y_{k+1}-0.8\right) x_{k} \text {, } \\
& \frac{y_{k+1}-y_{k}}{\frac{1}{0.6}\left[1-e^{\frac{-0.6}{0.5}\left[(t+h)^{0.5}-t^{0.5}\right]}\right]}=1-0.6 y_{k}-x_{k+1} x_{k} \\
& \frac{z_{k+1}-z_{k}}{\left[1-e^{\frac{-1}{0.6}\left[(t+h)^{0.6}-t^{0.6}\right]}\right]}=-x_{k}-z_{k} \\
& \frac{x_{k+1}-x_{k}}{\frac{1}{0.3}\left[(t+h)^{0.3}-t^{0.3}\right]}=z_{k}+\left(y_{k+1}-0.8\right) x_{k}, \\
& \frac{y_{k+1}-y_{k}}{\frac{1}{0.5}\left[(t+h)^{0.5}-t^{0.5}\right]}=1-0.6 y_{k}-x_{k+1} x_{k} \text {, } \\
& \frac{z_{k+1}-z_{k}}{\frac{1}{0.6}\left[(t+h)^{0.6}-t^{0.6}\right]}=-x_{k}
\end{aligned}
$$$$
\text { are compared to (4.5), obtained by the CEFD method, }
$$$$
x_{k+1}-x_{k}+\frac{h^{0.3}}{0.3}\left(z_{k}+\left(y_{k}-0.8\right) x_{k}\right) \text {, }
$$$$
y_{k+1}=y_{k}+\frac{h^{0.5}}{0.5}\left(1-0.6 y_{k}-x_{k} x_{k}\right) \text {, }
$$$$
z_{k+1}=z_{k}+\frac{h^{0.6}}{0.6}\left(x_{k}-z_{k}\right) \text {. }
$$

While bifurcations can be seen in the CEFD model, they are absent from the results of the ESDDFD models.

\section{Figures 4.1}



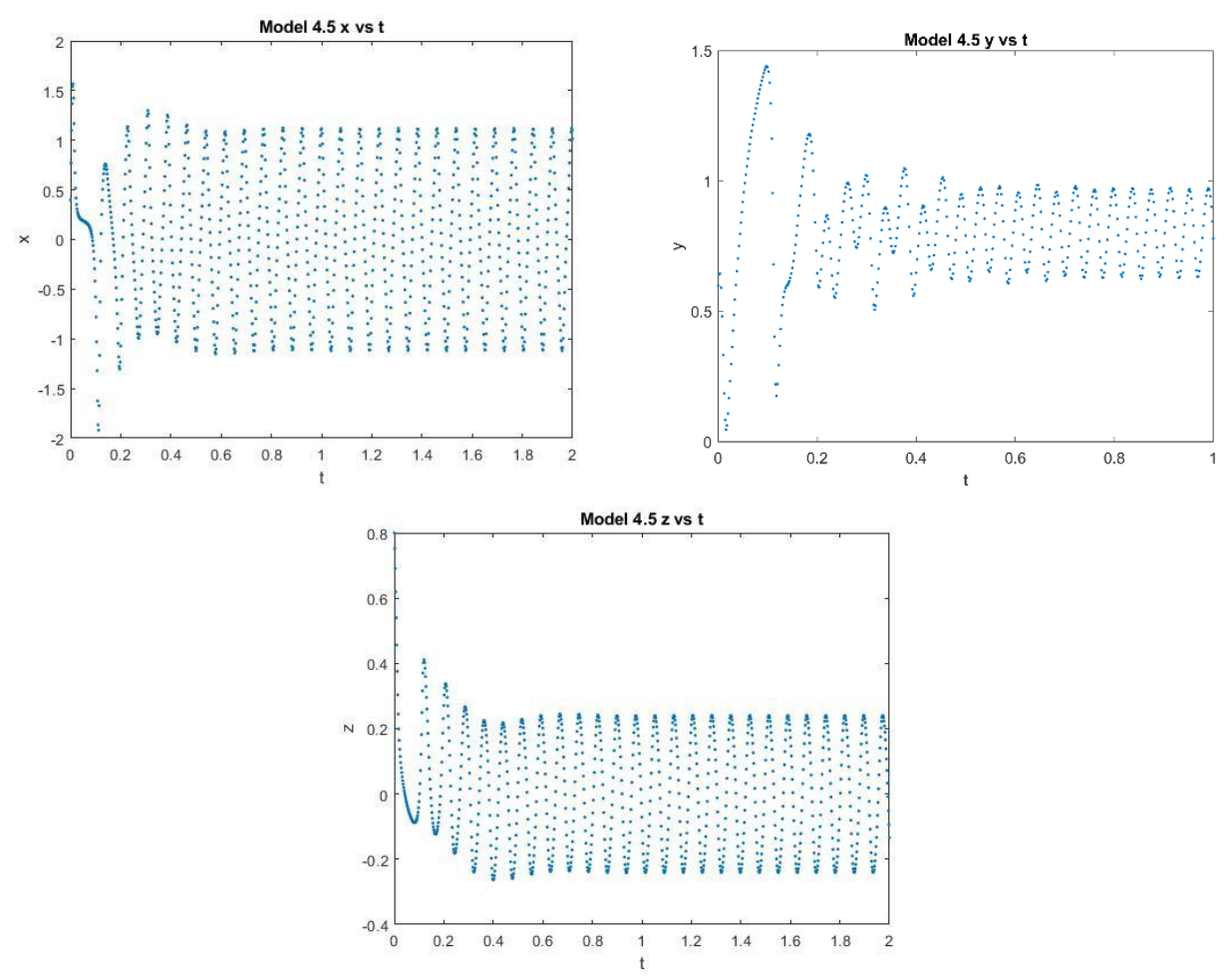

Figures 4.1(a). CEFD model (4.5) profiles of $x, y z$
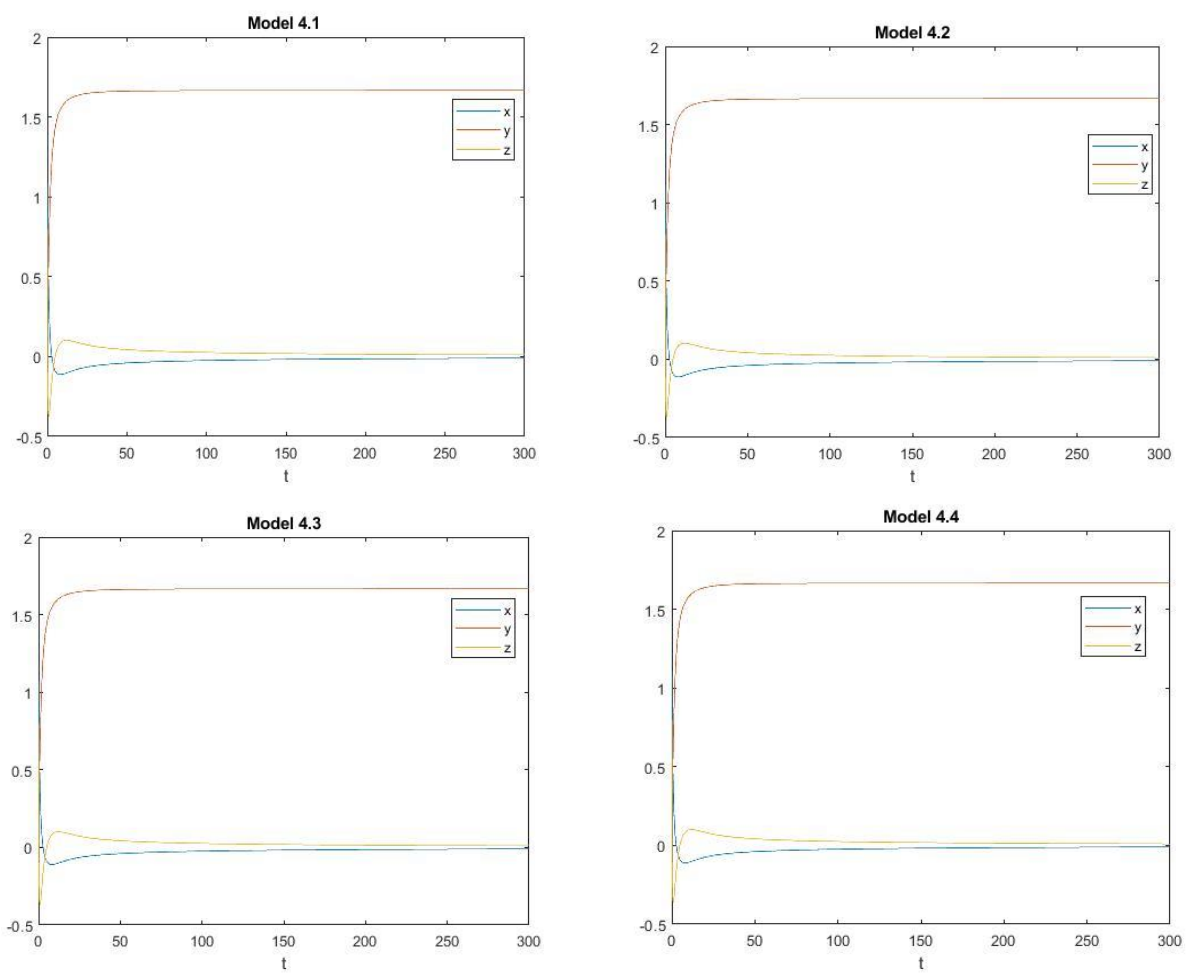

Figures 4.1(b). Profiles of $x, y, z$ for each model (4.1) through (4.4) 


\subsection{Five-dimensional systems comparison; Varying $\alpha 5, k$, and $p$}

For this case experiments performed in [Xin et al., 2019] are performed with the same parameters for the models (3.4), obtained by the ESDDFD method, for the various cases and values of $\left(\alpha_{5}, k, p\right)$ used in [Xin et al], the CEFD model (???)

$$
\begin{aligned}
& x_{k+1}=x_{k}+\frac{h^{0.3}}{0.3}\left(z_{k}+\left(y_{k}-0.8\right) x_{k}+k\left(w_{k}-p u_{k}\right)\right) \\
& y_{k+1}=y_{k}+\frac{h^{0.5}}{0.5}\left(1-0.6 y_{k}-x_{k} x_{k}+k\left(w_{k}-p u_{k}\right)\right) \\
& z_{k+1}=z_{k}-\frac{h^{0.6}}{0.6}\left(x_{k}+z_{k}-k\left(w_{k}-p u_{k}\right)\right) \\
& w_{k+1}=w_{k}-\frac{h^{0.24}}{0.24} 2 x_{k} y_{k} z_{k} \\
& u_{k+1}=u_{k}+\frac{h^{\alpha_{5}}}{\alpha_{5}} k\left(w_{k}-p u_{k}\right)
\end{aligned}
$$

are compared to the following four models (3.4), respectively MCEFD, ESDDFD1, ESDDFD2, ESDDFD3, obtained by the ESDDFD and NSFD methods:

$$
\begin{aligned}
& \frac{x_{k+1}-x_{k}}{\frac{1}{0.3}\left[(t+h)^{0.3}-t^{0.3}\right]}=z_{k}+\left(y_{k}-0.8\right) x_{k}+k\left(w_{k}-p u_{k}\right) \\
& \frac{y_{k+1}-y_{k}}{\frac{1}{0.5}\left[(t+h)^{0.5}-t^{0.5}\right]}=1-0.6 y_{k}-x_{k} x_{k}+k\left(w_{k}-p u_{k}\right) \text {, } \\
& \frac{z_{k+1}-z_{k}}{\frac{1}{0.6}\left[(t+h)^{0.6}-t^{0.6}\right]}=-x_{k}-z_{k}+k\left(w_{k}-p u_{k}\right) \text {, } \\
& \frac{w_{k+1}-w_{k}}{\frac{1}{0.24}\left[(t+h)^{0.24}-t^{0.24}\right]}=-x_{k} y_{k}\left(z_{k}+z_{k}\right) \\
& \frac{u_{k+1}-u_{k}}{\frac{1}{\alpha_{5}}\left[(t+h)^{\alpha_{5}}-t^{\alpha_{5}}\right]}=k\left(w_{k}-p u_{k}\right) \text {, } \\
& \frac{x_{k+1}-x_{k}}{\frac{1}{0.8}\left[1-e^{\frac{-0.8}{0.3}\left[(t+h)^{0.3}-t^{0.3}\right]}\right]}=z_{k}+\left(y_{k}-0.8\right) x_{k}+k\left(w_{k}-p u_{k}\right) \\
& \frac{y_{k+1}-y_{k}}{\frac{1}{0.6}\left[1-e^{\frac{-0.6}{0.5}\left[(t+h)^{0.5}-t^{0.5}\right]}\right]}=1-0.6 y_{k}-x_{k} x_{k}+k\left(w_{k}-p u_{k}\right) \text {, } \\
& \frac{z_{k+1}-z_{k}}{\left[1-e^{\frac{-1}{0.6}\left[(t+h)^{0.6}-t^{0.6}\right]}\right]}=-x_{k}-z_{k}+k\left(w_{k}-p u_{k}\right) \text {, } \\
& \frac{w_{k+1}-w_{k}}{\left[1-e^{\frac{-1}{0.24}\left[(t+h)^{0.24}-t^{0.24}\right]}\right]}=-x_{k} y_{k}\left(z_{k}+z_{k}\right) \\
& \frac{u_{k+1}-u_{k}}{\frac{1}{\mathrm{kp}}\left[1-e^{\frac{-k p}{\alpha_{5}}\left[(t+h)^{\alpha_{5}-t^{\alpha}}\right]}\right]}=k\left(w_{k}-p u_{k}\right)
\end{aligned}
$$




$$
\begin{aligned}
& \frac{x_{k+1}-x_{k}}{\frac{1}{0.3}\left[(t+h)^{0.3}-t^{0.3}\right]}=z_{k}+\left(y_{k+1}-0.8\right) x_{k}+k\left(w_{k}-p u_{k}\right) \\
& \frac{y_{k+1}-y_{k}}{\frac{1}{0.5}\left[(t+h)^{0.5}-t^{0.5}\right]}=1-0.6 y_{k}-x_{k+1} x_{k}+k\left(w_{k}-p u_{k}\right) \text {, } \\
& \frac{z_{k+1}-z_{k}}{\frac{1}{0.6}\left[(t+h)^{0.6}-t^{0.6}\right]}=-x_{k}-z_{k}+k\left(w_{k}-p u_{k}\right) \text {, } \\
& \frac{w_{k+1}-w_{k}}{\frac{1}{0.24}\left[(t+h)^{0.24}-t^{0.24}\right]}=-x_{k} y_{k+1}\left(z_{k}+z_{k+1}\right) \\
& \frac{u_{k+1}-u_{k}}{\frac{1}{\alpha_{5}}\left[(t+h)^{\alpha_{5}} t^{\alpha_{5}}\right]}=k\left(w_{k}-p u_{k}\right) \text {, } \\
& \frac{x_{k+1}-x_{k}}{\frac{1}{0.8}\left[1-e^{\frac{-0.8}{0.3}\left[(t+h)^{0.3}-t^{0.3}\right]}\right]}=z_{k}+\left(y_{k+1}-0.8\right) x_{k}+k\left(w_{k}-p u_{k}\right) \\
& \frac{y_{k+1}-y_{k}}{\frac{1}{0.6}\left[1-e^{\frac{-0.6}{0.5}\left[(t+h)^{0.5}-t^{0.5}\right]}\right]}=1-0.6 y_{k}-x_{k+1} x_{k}+k\left(w_{k}-p u_{k}\right) \text {, } \\
& \frac{z_{k+1}-z_{k}}{\left[1-e^{\frac{-1}{0.6}\left[(t+h)^{0.6}-t^{0.6}\right]}\right]}=-x_{k}-z_{k}+k\left(w_{k}-p u_{k}\right) \\
& \frac{w_{k+1}-w_{k}}{\left[1-e^{\frac{-1}{0.24}\left[(t+h)^{0.24}-t^{0.24}\right]}\right]}=-x_{k} y_{k+1}\left(z_{k}+z_{k+1}\right) \\
& \frac{u_{k+1}-u_{k}}{\frac{1}{\mathrm{kp}}\left[1-e^{\frac{-k p}{\alpha_{5}}\left[(t+h)^{\alpha_{5}-t^{\alpha}}\right]}\right]}=k\left(w_{k}-p u_{k}\right)
\end{aligned}
$$

4.2.1 Varying $\alpha 5$ with fixed $k=2$ and $p=1$ and $\alpha_{5} \in[0.232,0.328]$.

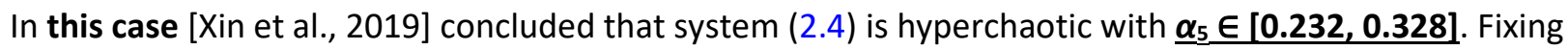
$\alpha 5=0.24$, a set of two positive Lyapunov exponents and three negative Lyapunov exponents. Profiles for $x, y, z, w$ and $u$, when $\alpha_{5}=0.232$ for model (4.6) are given below. For each model (4.7) through (4.10) a graph of the five variables is given using the same step size and parameter values. These models produce identical graphs which differ significantly from the graphs for model (4.6). The Bifurcation tests for the ESDDFD model (3.4) are performed with the same parameters. The bifurcations diagrams for $x, z$ and $u$ for models (4.6) through (4.10) are reproduced for $h=0.002$. 


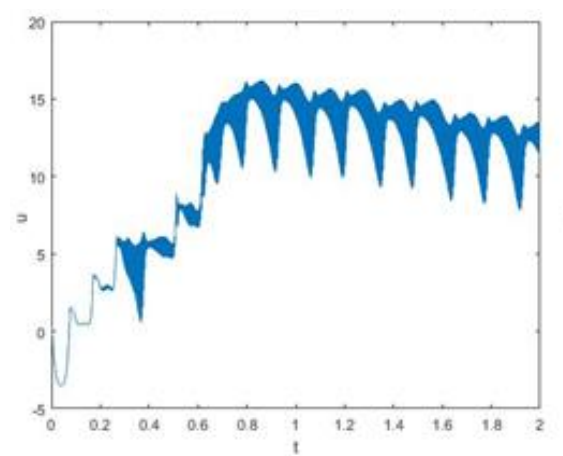

(i)

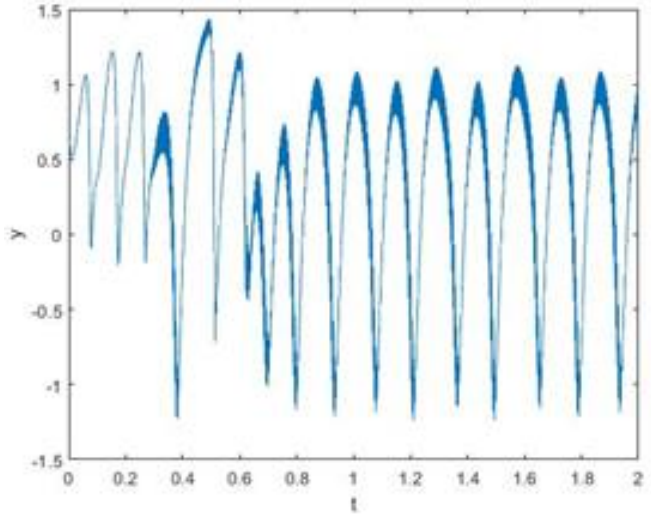

(iv)

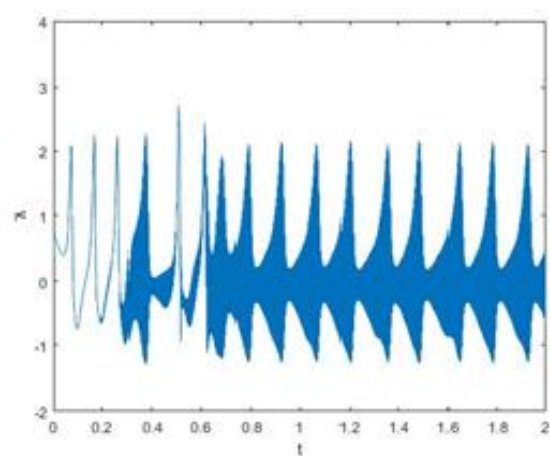

(ii)

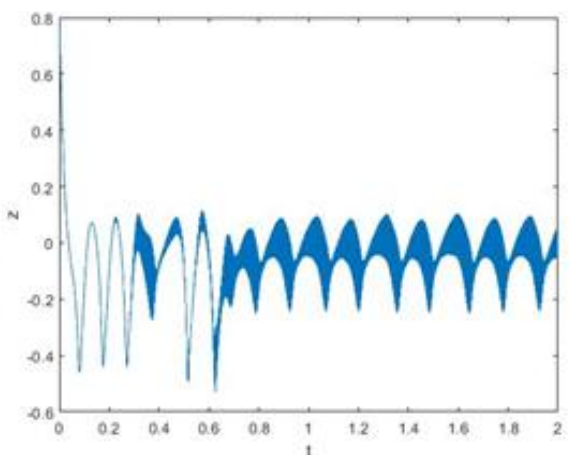

(iii)

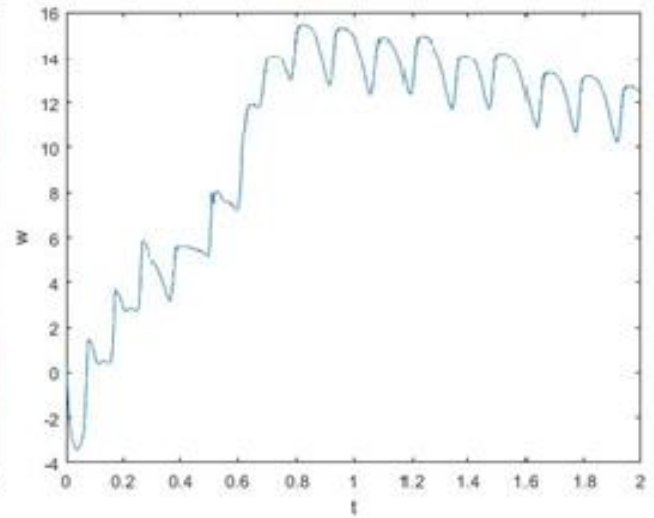

(v)

Figures 4.2.1(a). CEFD model (4.6) profiles of $(i) u,(i i) x$, (iii)z, (iv) $y,(v) w$, at $k=2, p=1, \alpha_{5}=0.232$
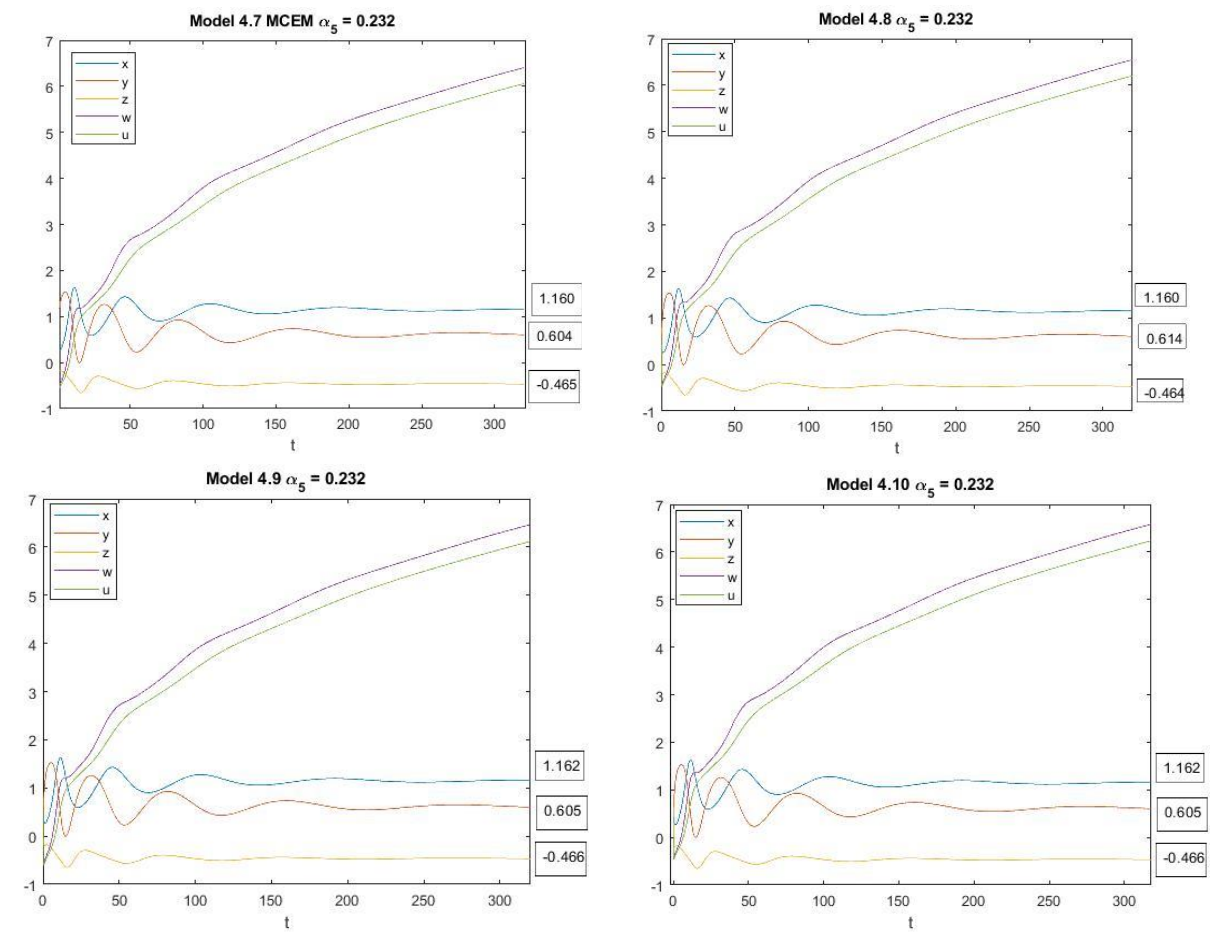

Figures 4.2.1(b). Models (4.7), (4.8), (4.9) and (4.10) profiles of $x, y, z, w$ and $u$ at $k=2, p=1, \alpha_{5}=0.232$ 

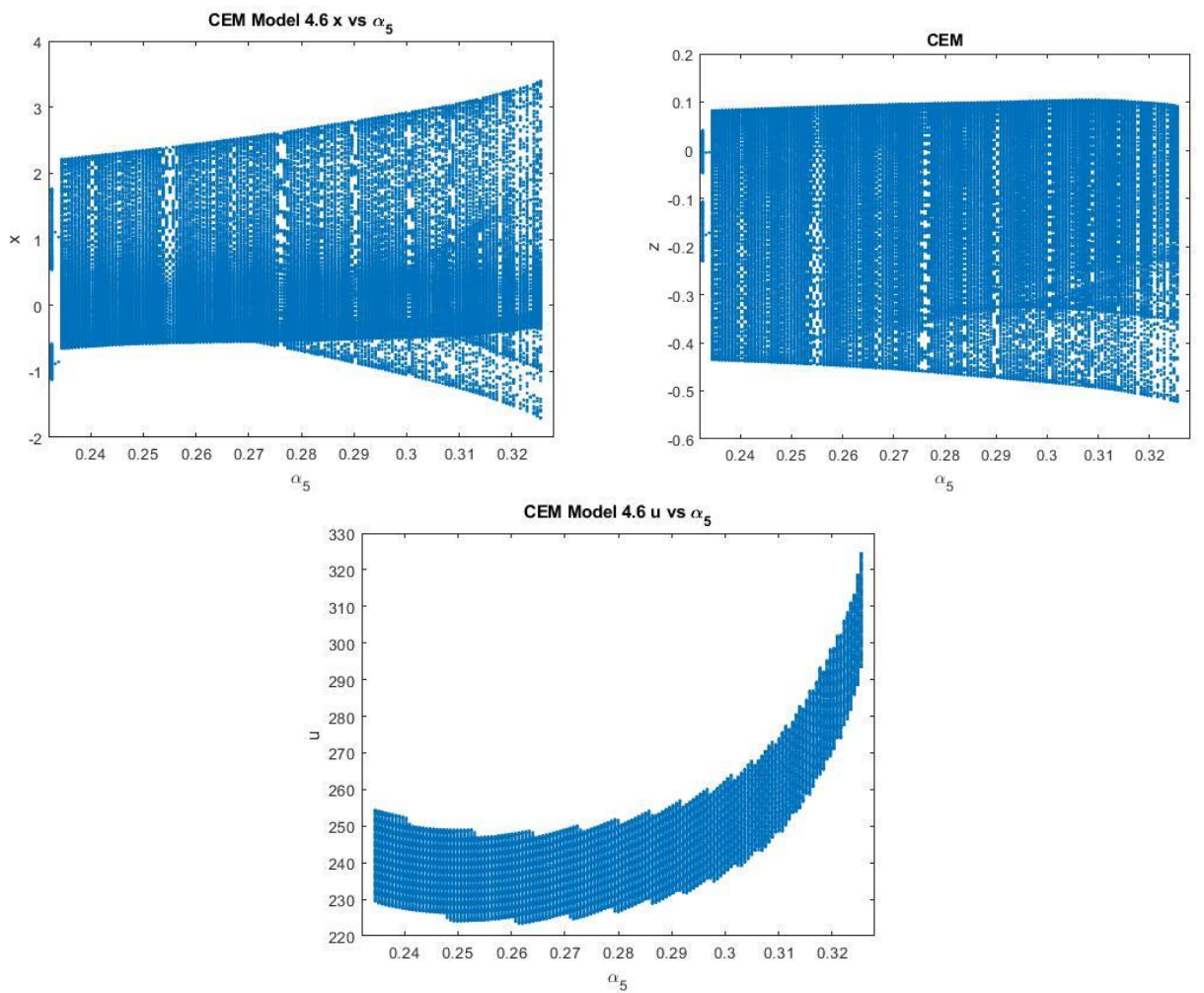

Figures 4.2.1(c). CEFD model (4.6) bifurcation of $x, z, u$ versus $\alpha_{5}$ for $h=0.002$
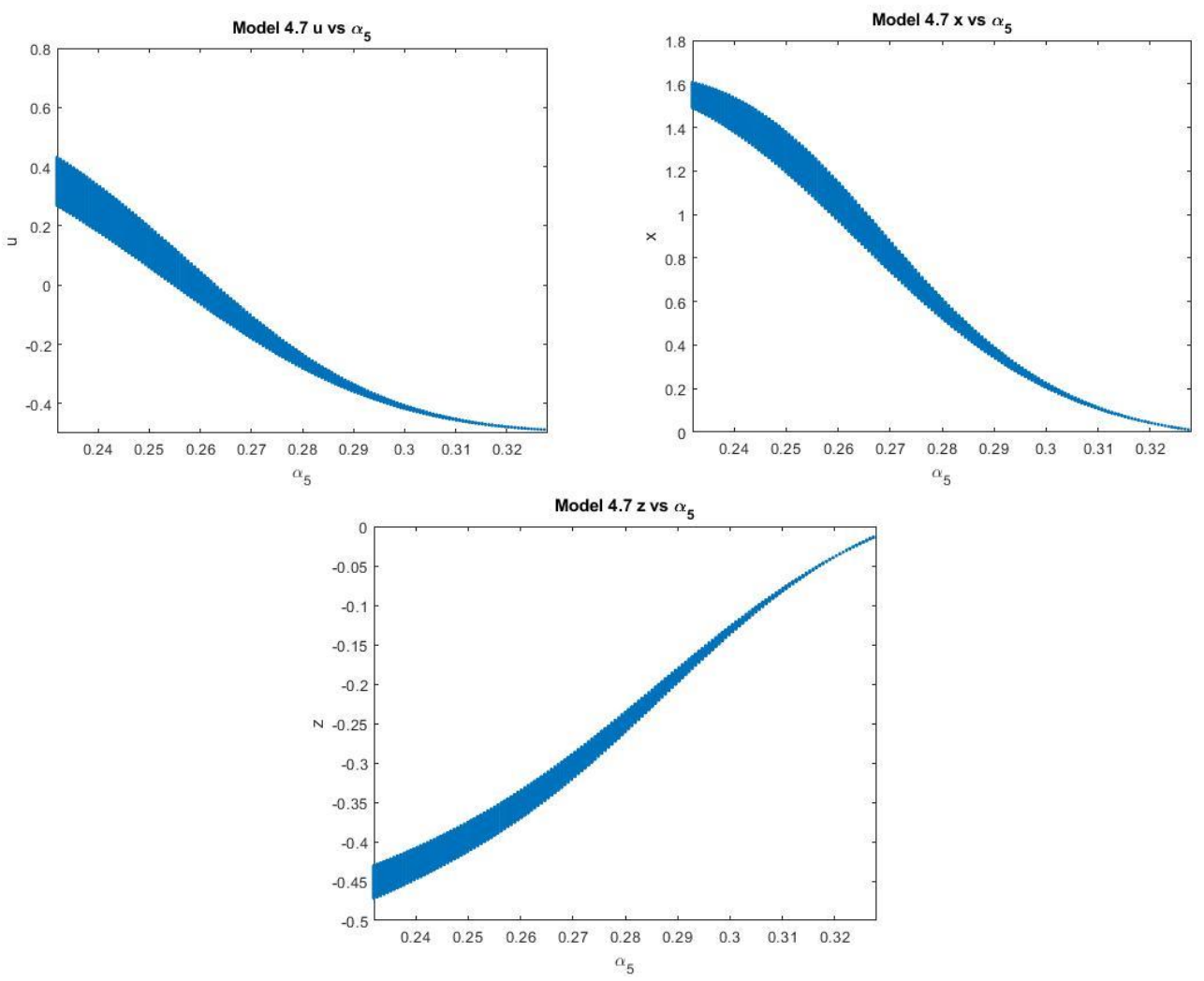

Figures 4.2.1(d). ESDDFD model (4.7), (i)uvs $\alpha_{5}$, (ii) $x$ vs $\alpha_{5}$, (iii)z $v s \alpha_{5}$, at $k=2, p=1, \alpha_{5} \in[0.232,0.328]$ 

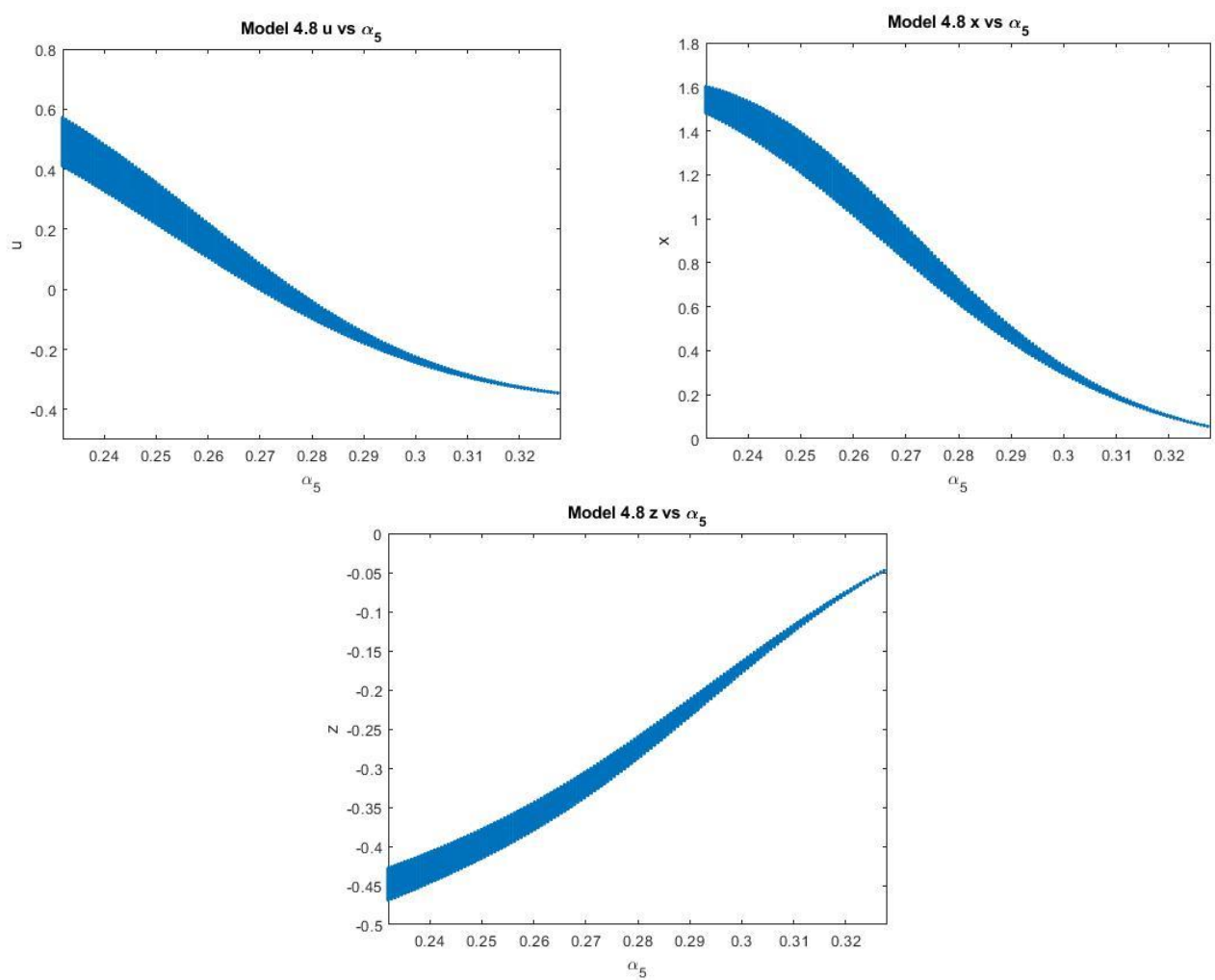

Figures 4.2.1(e). ESDDFD model (4.8), (i) $u$ vs $\alpha_{5}$, (ii) $x$ vs $\alpha_{5}$, (iii)z vs $\alpha_{5}$, at $k=2, p=1, \alpha_{5} \in[0.232,0.328]$
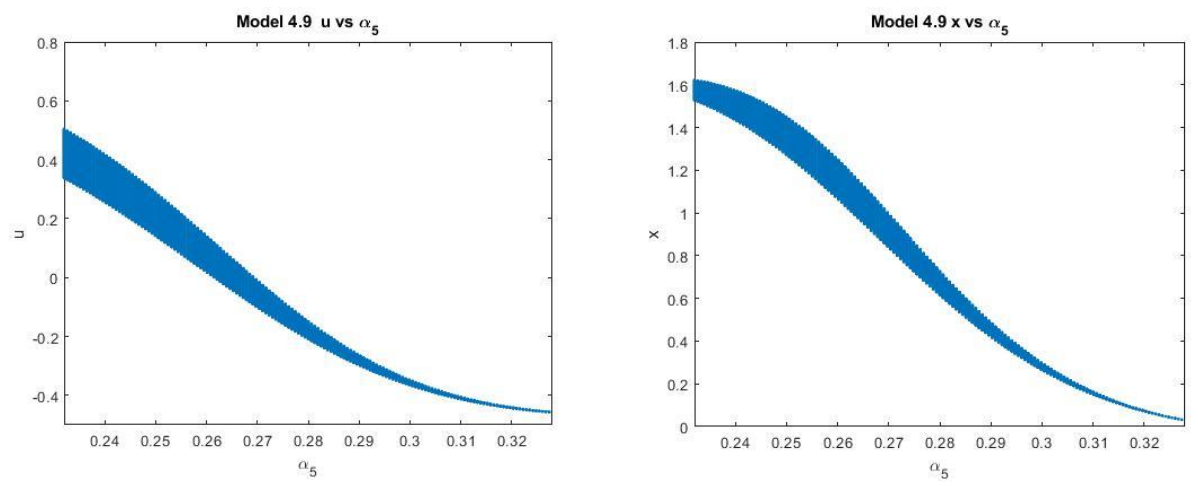


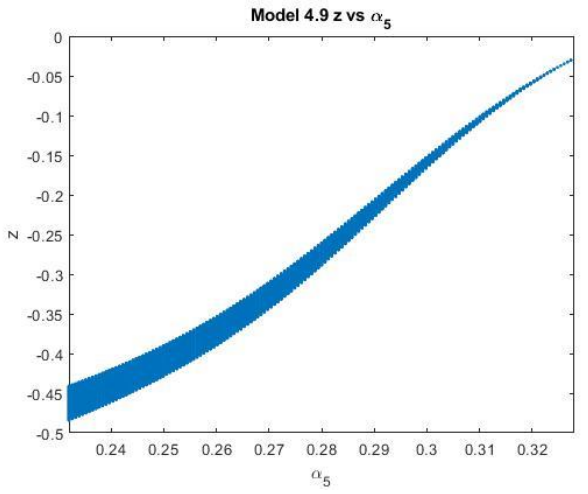

Figures 4.2.1(f). ESDDFD model (4.9), (i)u vs $\alpha_{5},(i i) x v s \alpha_{5}$, (iii)z vs $\alpha_{5}$, at $k=2, p=1, \alpha_{5} \in[0.232,0.328]$
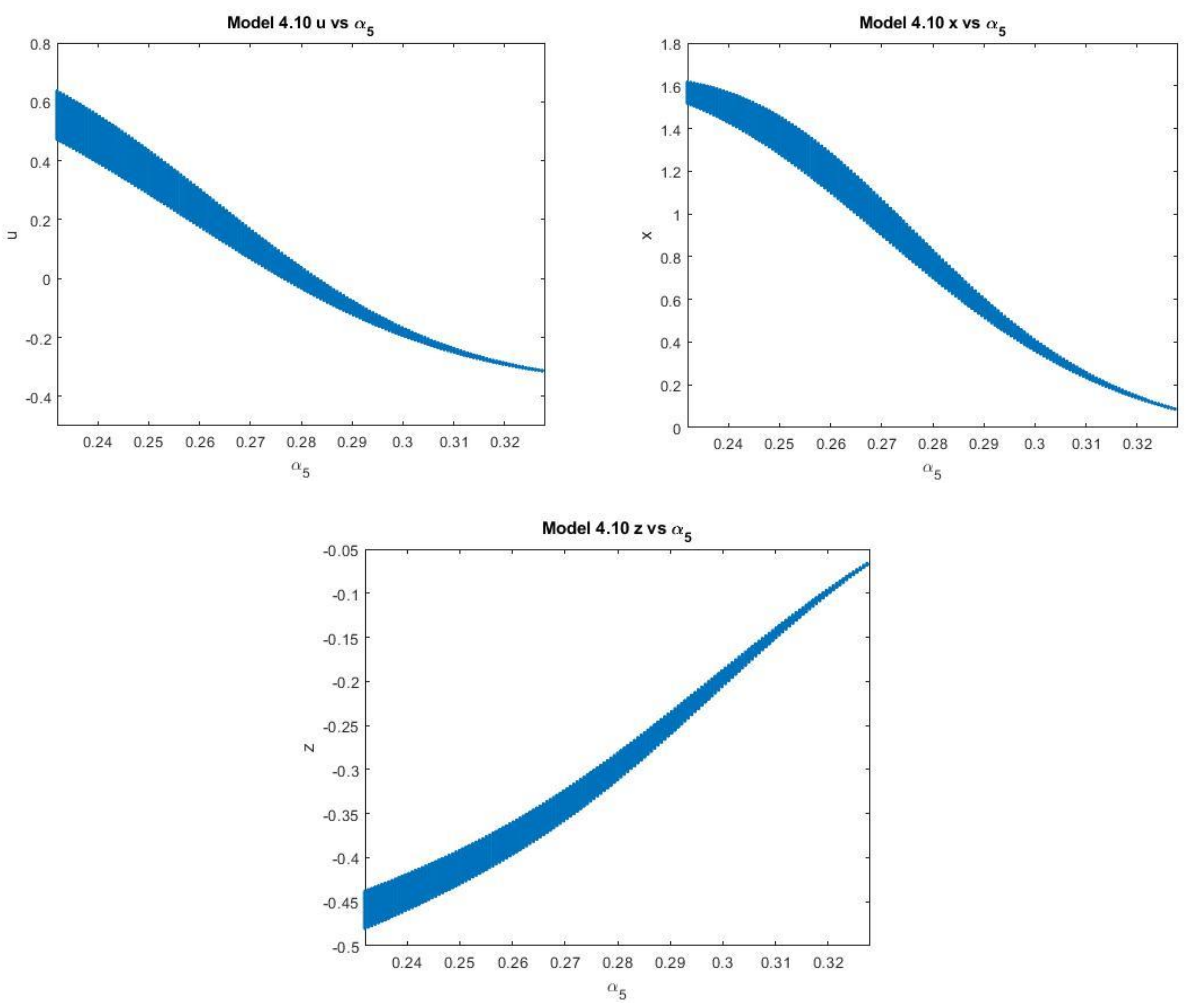

Figures 4.2.1(g). ESDDFD model (4.10), (i)u vs $\alpha_{5}$, (ii)x vs $\alpha_{5}$, (iii) z vs $\alpha_{5}$, at $k=2, p=1, \alpha_{5} \in[0.232,0.328]$ 

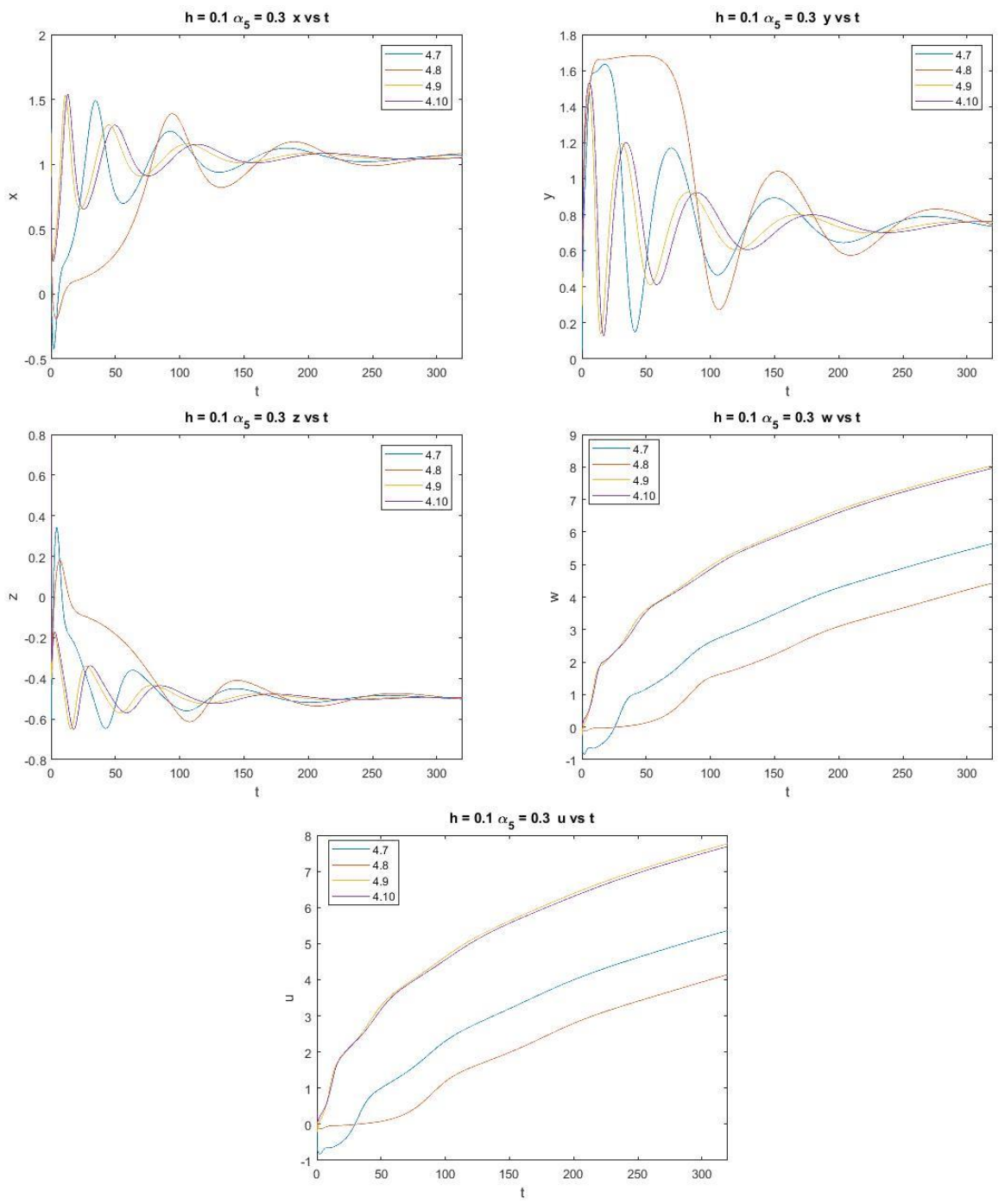

Figures 4.2.1(h) $h=0.1, \alpha_{5}=0.3 x, y, z, w, u$ for (4.7) through (4.10)

For step sizes above 0.003 CEFD fails. MCEFD fails for step sizes above 0.573 . The graphs below, using the same parameters as in Figure 4.2.1(b) with $h=1.0$, show the effect of larger step sizes on methods (4.8), (4.9) and (4.10). Note the differences in the early behavior between the methods, especially when compared with $\mathrm{h}=0.002$. 

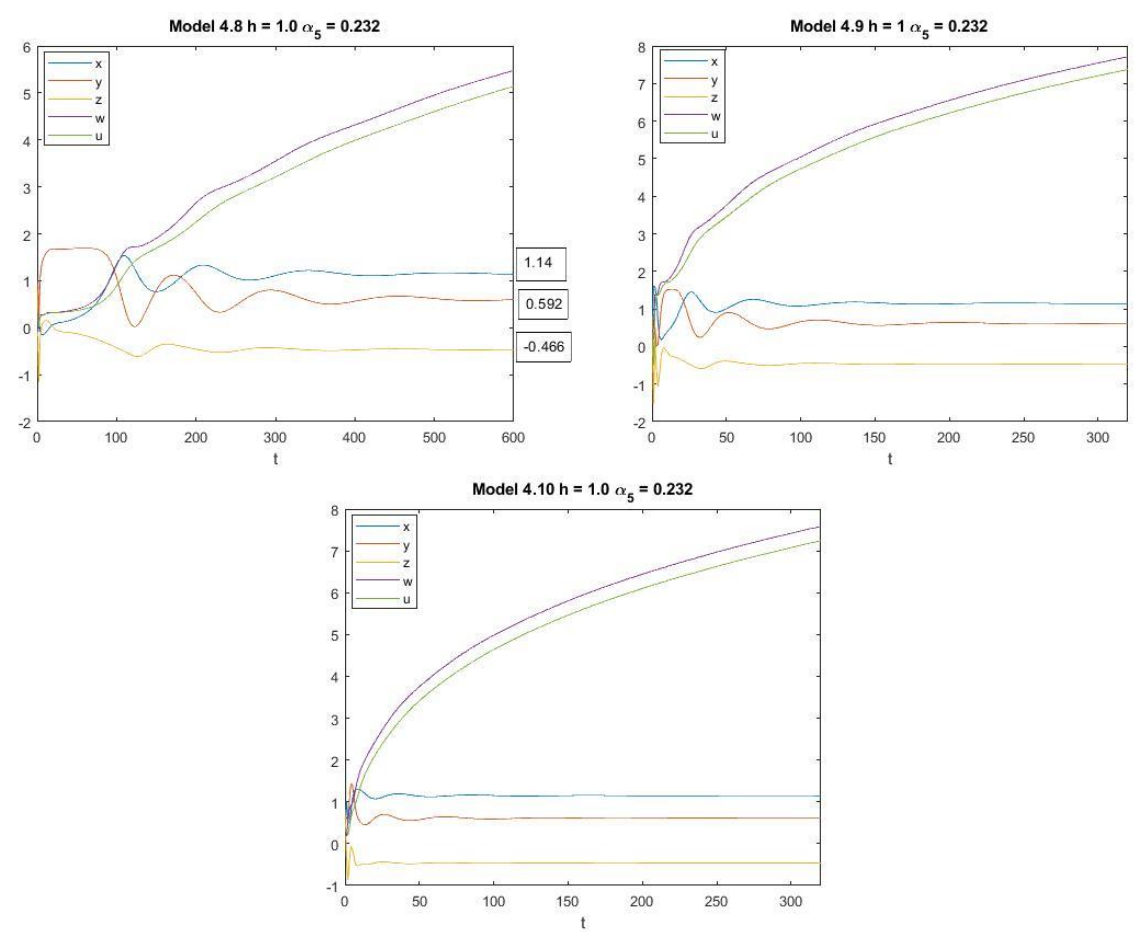

Figures 4.2.1(i) $h=1.0, \alpha_{5}=0.232 x, y, z, w, u$ for (4.8) through (4.10)

\subsubsection{Varying $p$ with fixed $k=2, \alpha 5=0.3$, and $p \in[1,2]$.}

In this case, [Xin et al., 2019] concluded that system (2.4) is hyperchaotic with $\boldsymbol{p} \in[\mathbf{1}, \mathbf{2}$ ]. Fixing $p=1$, a set of two positive Lyapunov exponents and three negative Lyapunov exponents was determined. Bifurcation tests for the ESDDFD model (3.4) are performed with the same parameters for the full discrete model (1.2).
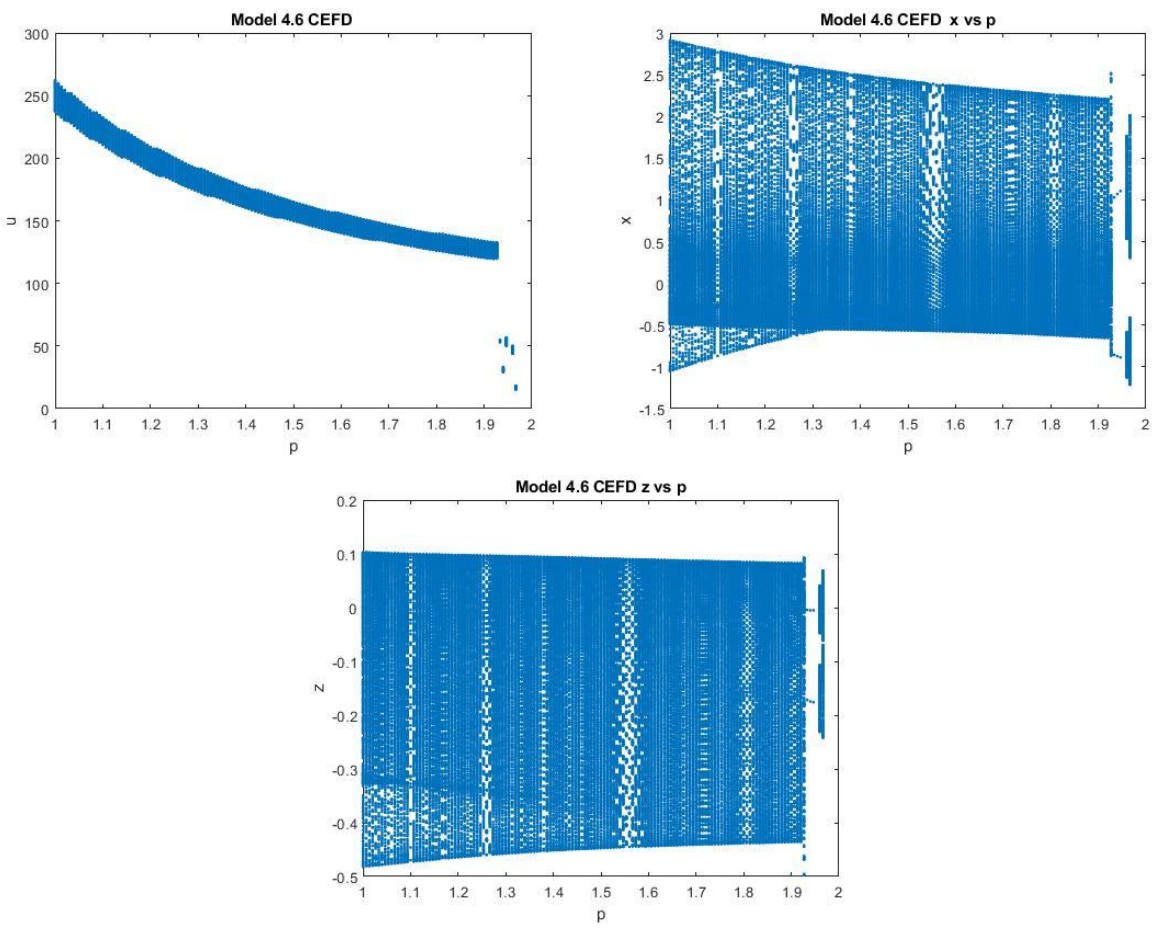
Figures 4.2.2(a). CEFD model (4.6) (i)u vs $\alpha_{5}$, (ii)x vs $\alpha_{5}$, (iii)z vs $\alpha_{5}$, at $k=2, \alpha_{5}=0.3, p \in[1,2]$
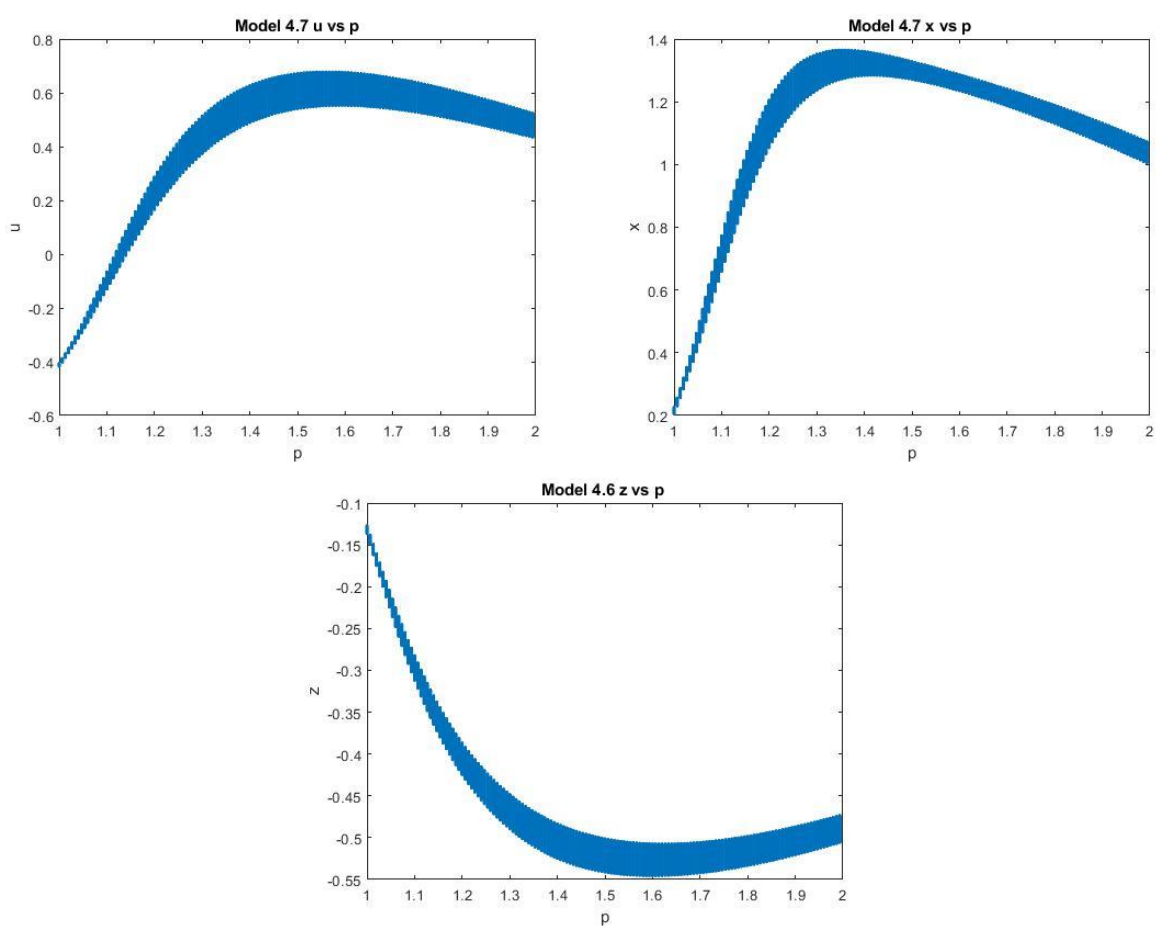

Figures 4.2.2(b). MCEFD model (4.7) (i)u vs $\alpha_{5}$, (ii) $x$ vs $\alpha_{5}$, (iii)z vs $\alpha_{5}$, at $k=2, \alpha_{5}=0.3, p \in[1,2]$
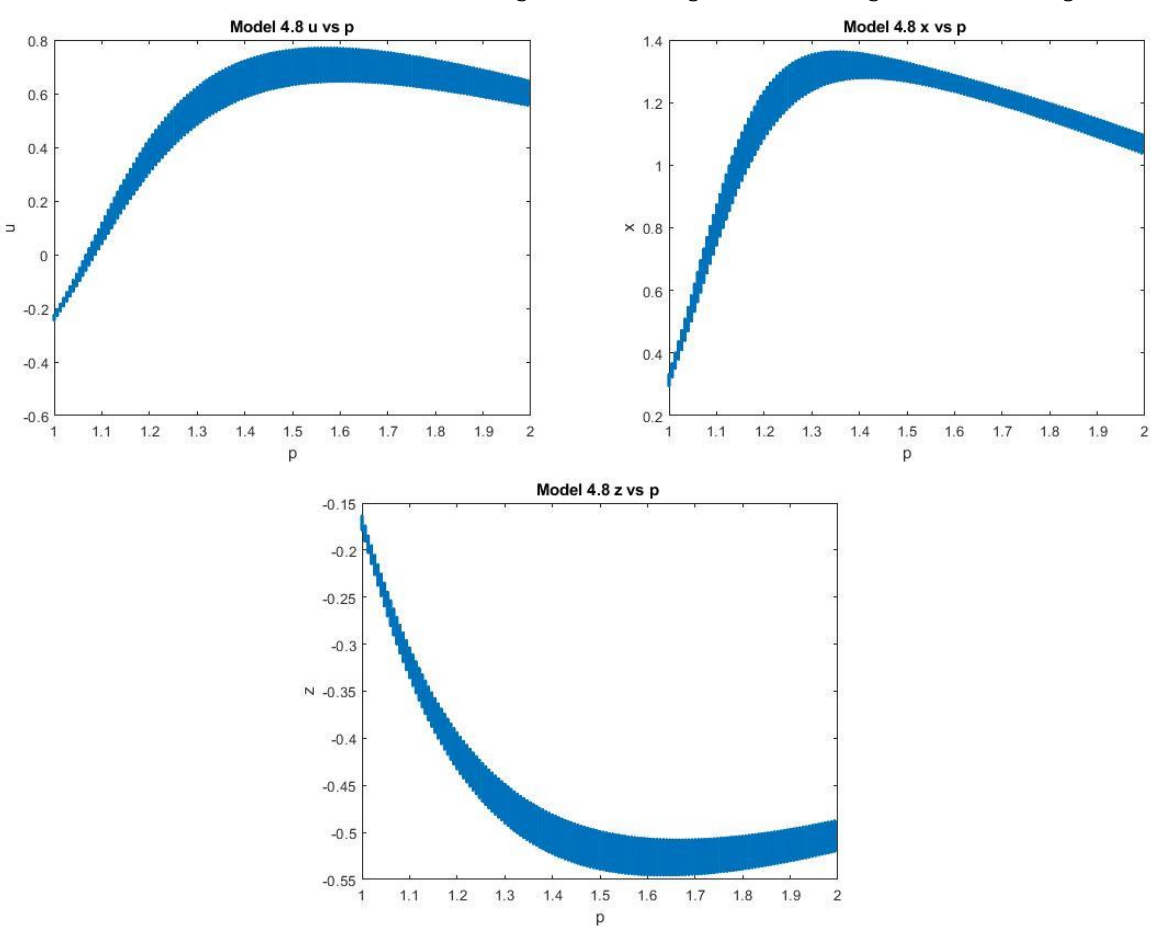

Figures 4.2.2(c). ESDDFD1 model (4.8) (i)u vs $\alpha_{5}$, (ii) $x$ vs $\alpha_{5}$, (iii)z vs $\alpha_{5}$, at $k=2, \alpha_{5}=0.3, p \in[1,2]$ 

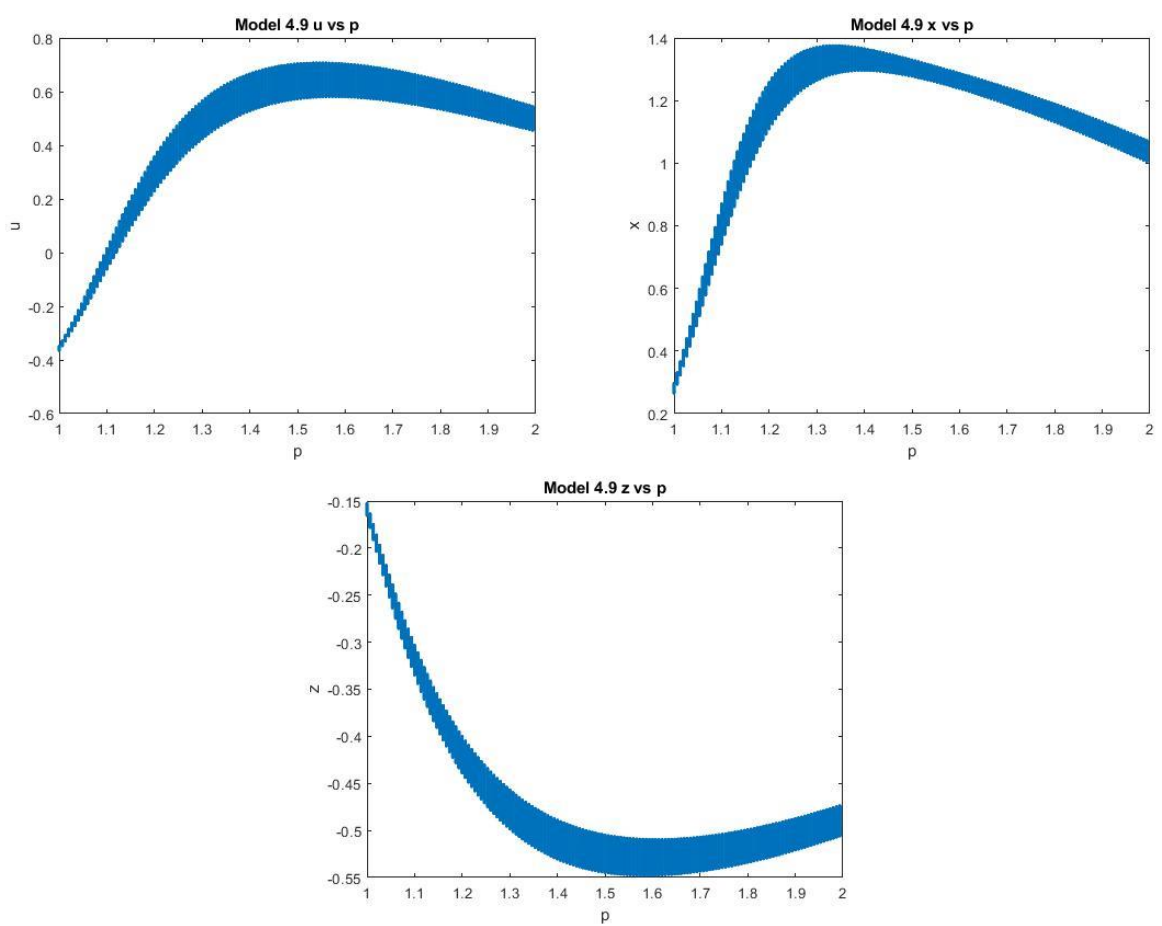

Figures 4.2.2(d). ESDDFD2 model (4.9) (i)u vs $\alpha_{5}$, (ii) $x$ vs $\alpha_{5}$, (iii) z vs $\alpha_{5}$, at $k=2, \alpha_{5}=0.3, p \in[1,2]$
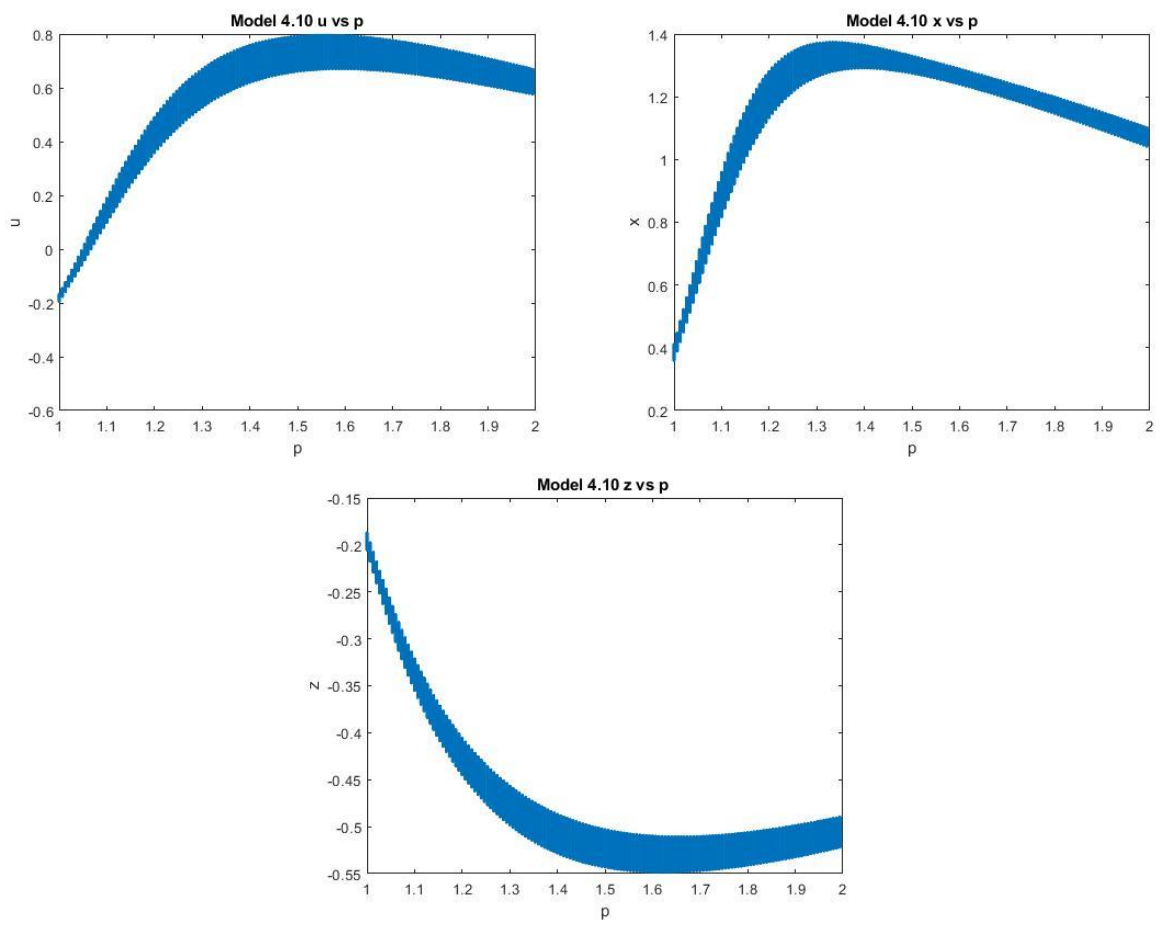

Figures 4.2.2(e). ESDDFD2 model (4.10) (i)u vs $\alpha_{5}$, (ii) $x$ vs $\alpha_{5}$, (iii)z vs $\alpha_{5}$, at $k=2, \alpha_{5}=0.3, p \in[1,2]$ 

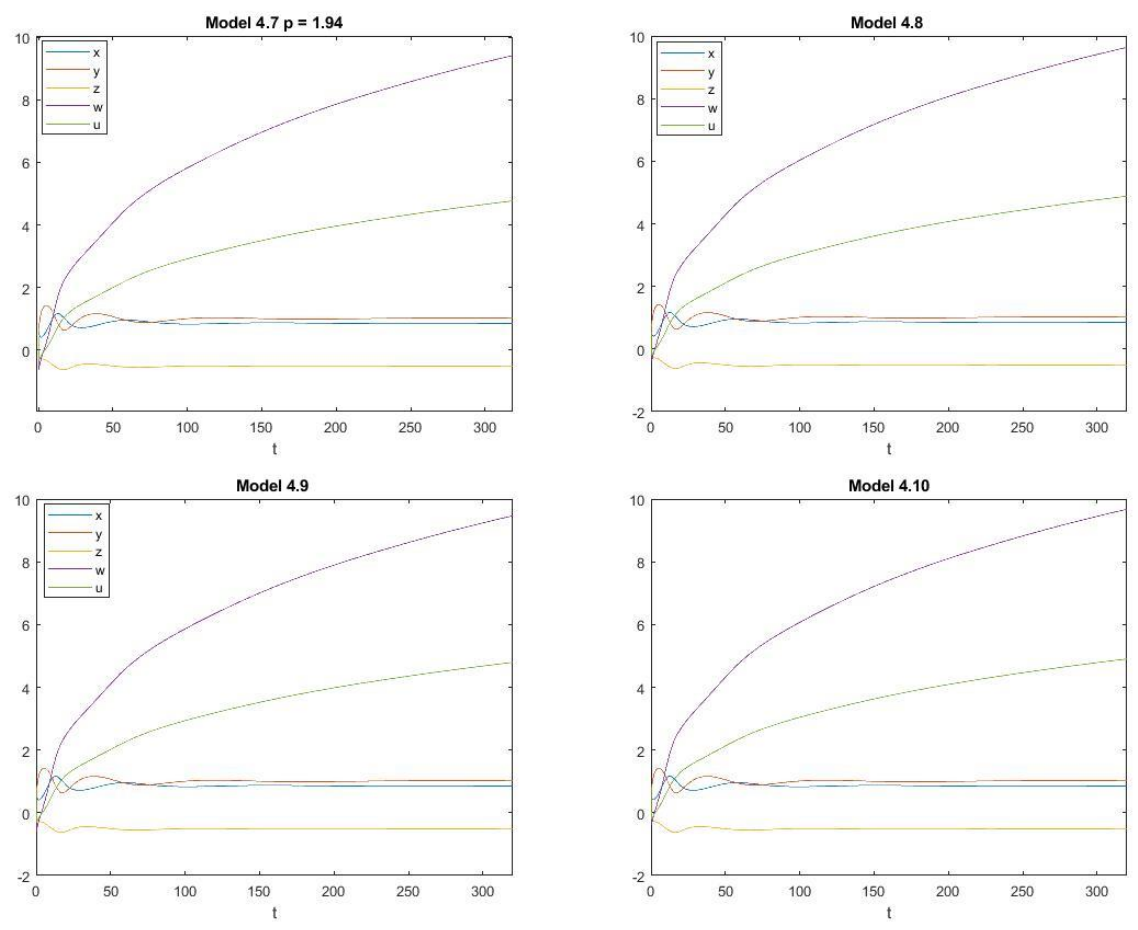

Figures 4.2.2(f). Models (4.7), (4.8), (4.9) and (4.10) profiles of $x, y, z, w$ and $u$ at $k=2, p=1.94, \alpha_{5}=0.3$

4.2.3 Varying $k$ with fixed $p=1$ and $\alpha 5=0.3$ with $k \in[1.5,2.5]$.

In this case [Xin et al., 2019] concluded that system (2.4) is hyperchaotic with $\boldsymbol{k} \in[\mathbf{1 . 5}, \mathbf{2 . 5}$. Fixing $k=1.5$, a set of two positive Lyapunov exponents and three negative Lyapunov exponents were determined. Bifurcation tests for the ESDDFD model (3.4) are performed with the same parameters for the full discrete model (1.2). 

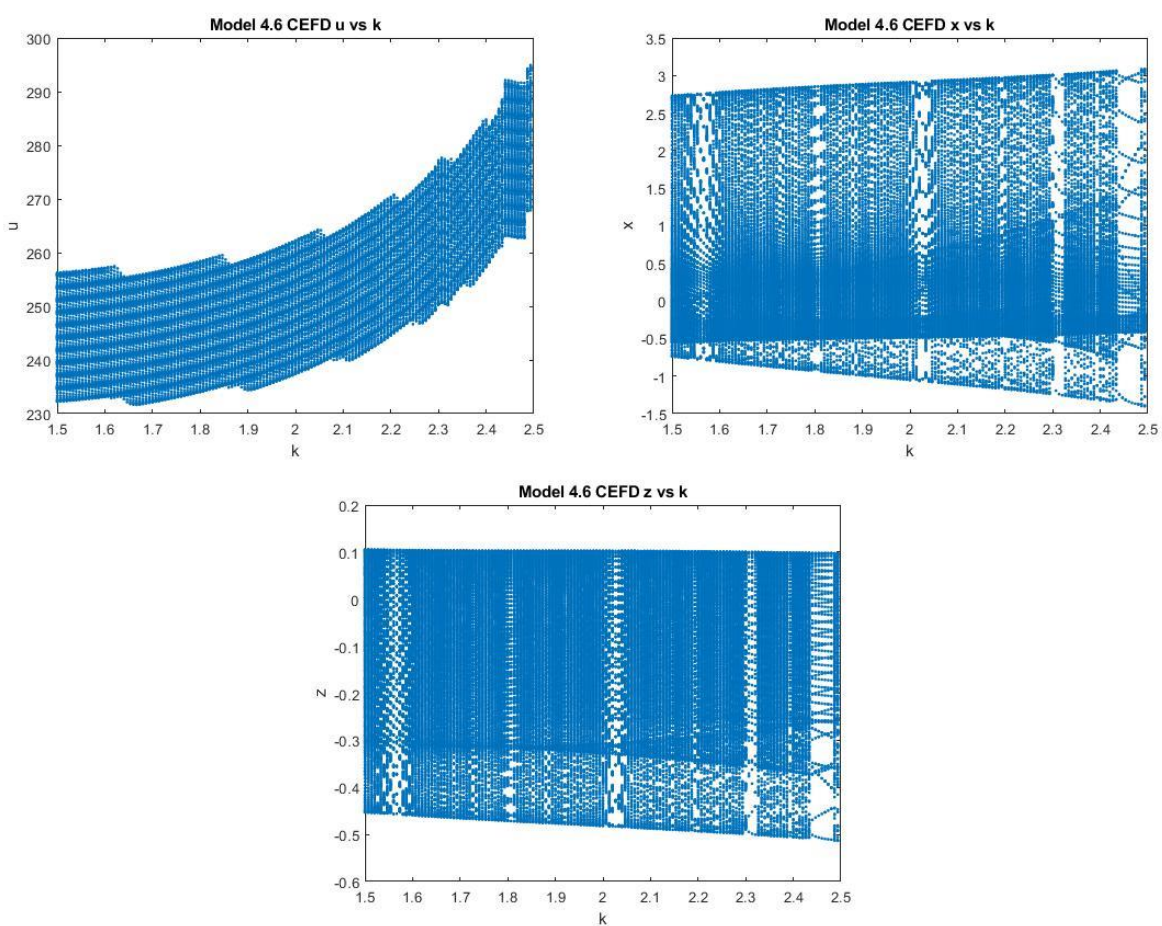

Figures 4.2.3(a). CEFD model (4.6) (i)u vs $\alpha_{5}$, (ii)x vs $\alpha_{5}$, (iii)z vs $\alpha_{5}$, at $p=1, \alpha_{5}=0.3, k \in[1.5,2.5]$
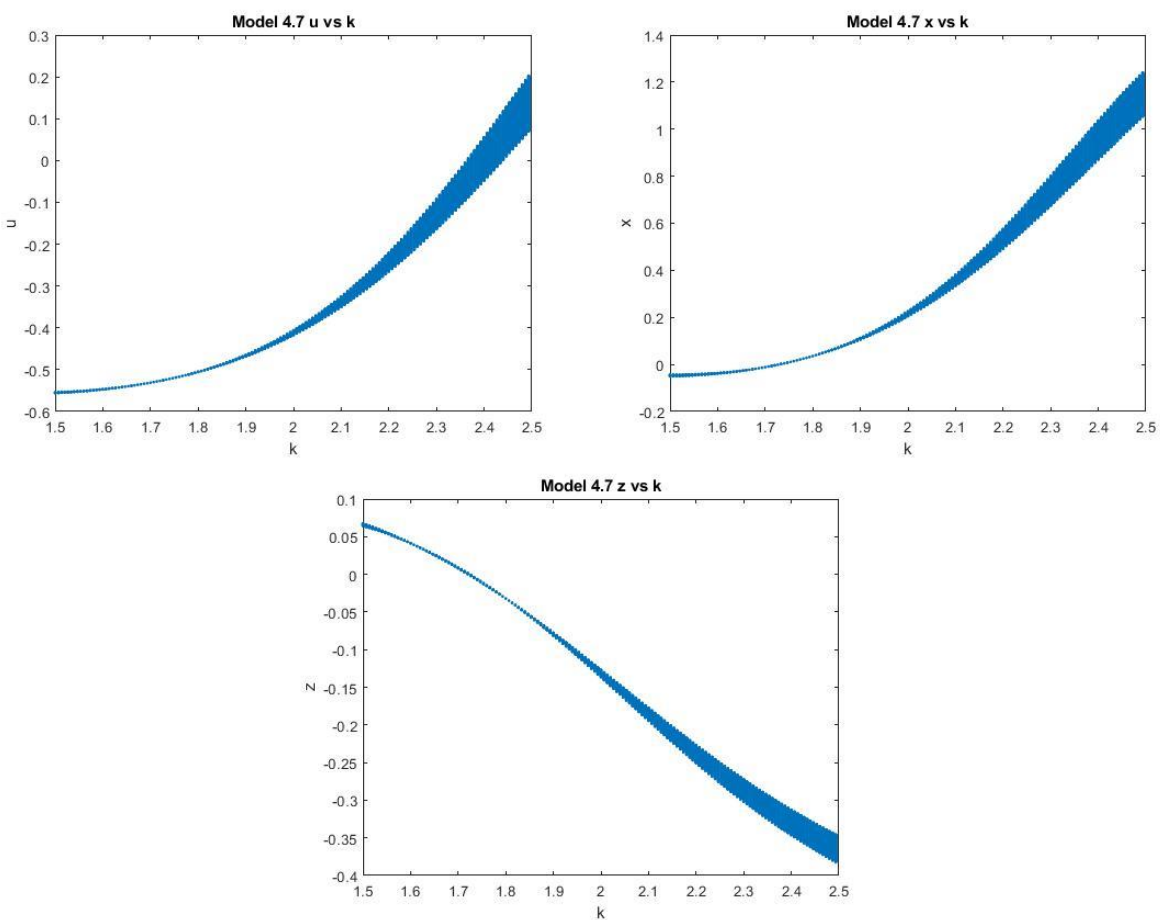

Figures 4.2.3(b). MCEFD model (4.7) (i)u vs $\alpha_{5}$, (ii) x vs $\alpha_{5}$, (iii)z vs $\alpha_{5}$, at $p=1, \alpha_{5}=0.3, k \in[1.5,2.5]$ 

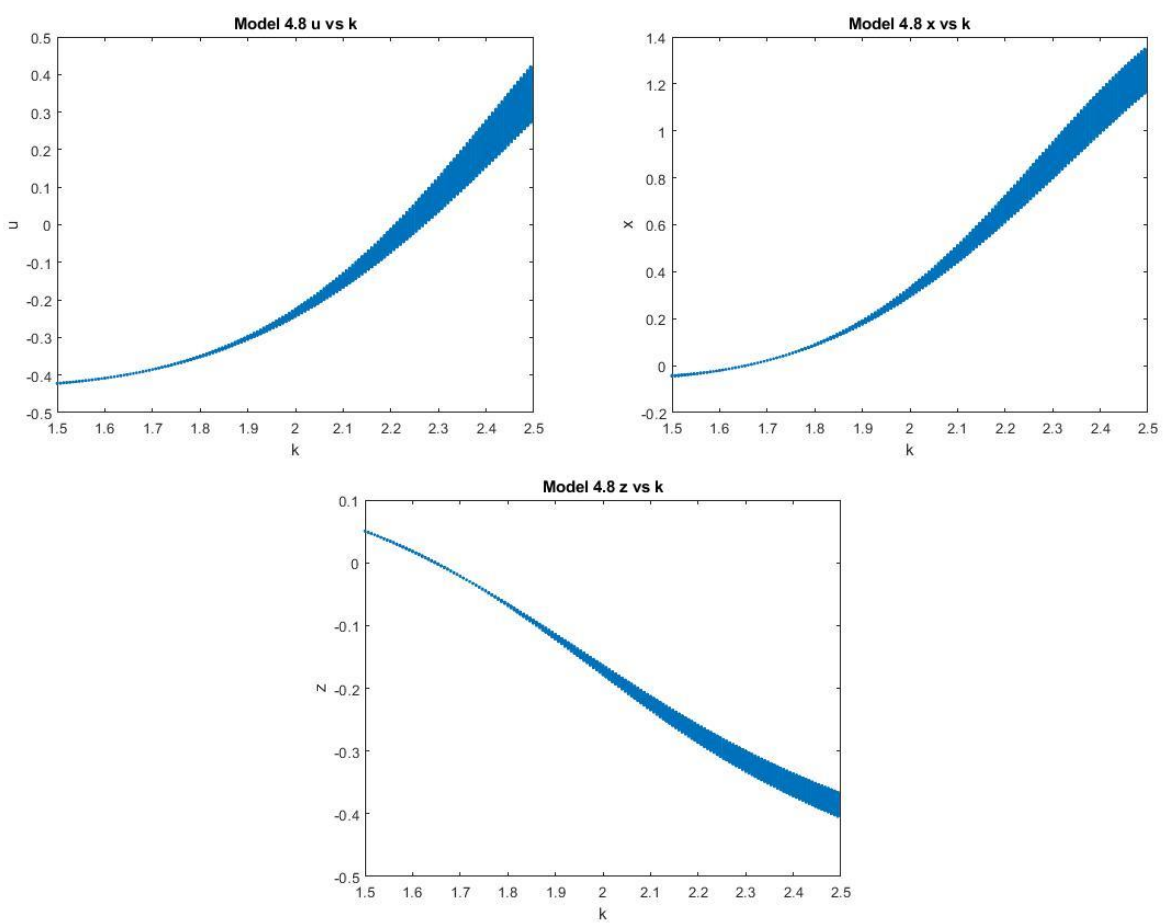

Figures 4.2.3(c). ESDDFD1 model (4.8) (i)u vs $\alpha_{5},(i i) x$ vs $\alpha_{5},(i i i) z$ vs $\alpha_{5}$, at $p=1, \alpha_{5}=0.3, k \in[1.5,2.5]$
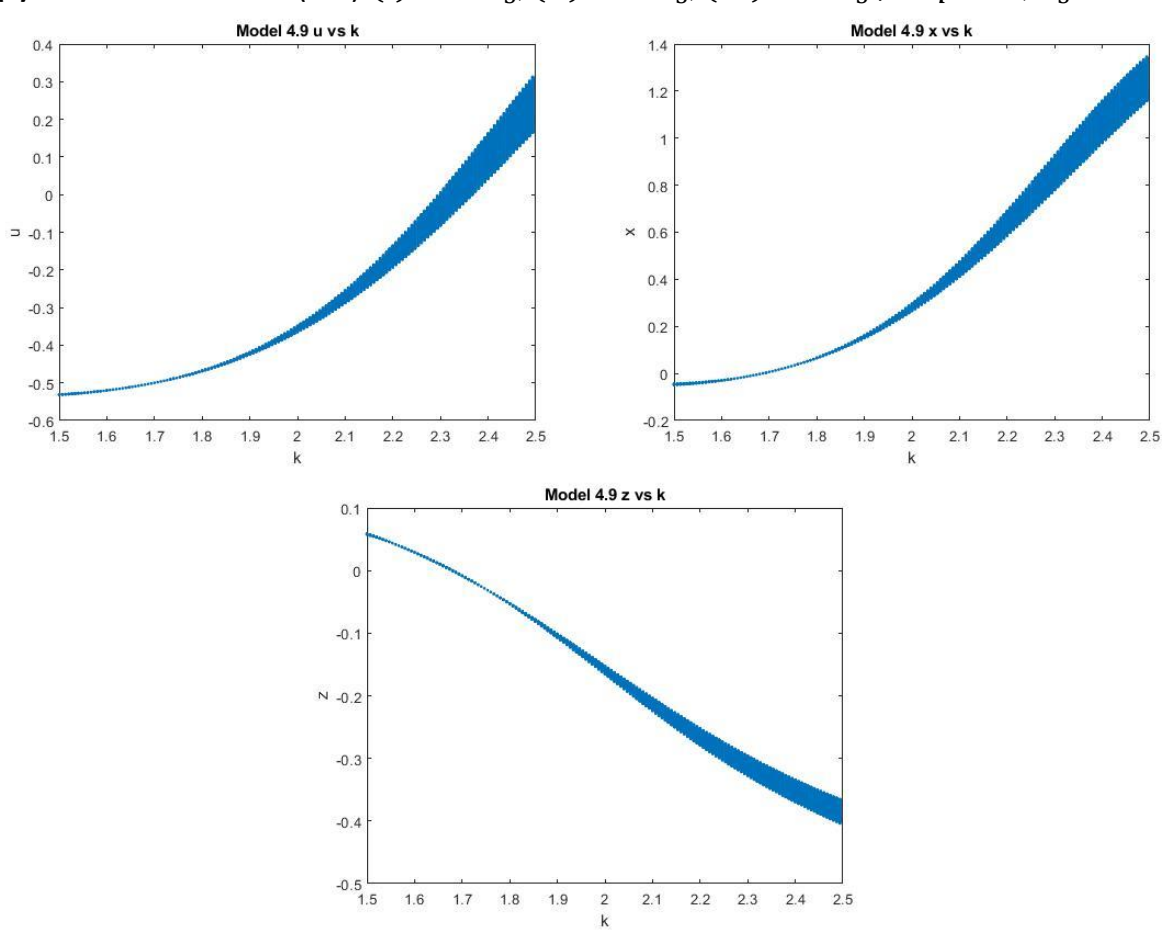

Figures 4.2.3(d). ESDDFD2 model (4.9) (i)u vs $\alpha_{5}$, (ii) $x$ vs $\alpha_{5}$, (iii) $z$ vs $\alpha_{5}$, at $p=1, \alpha_{5}=0.3, k \in[1.5,2.5]$ 

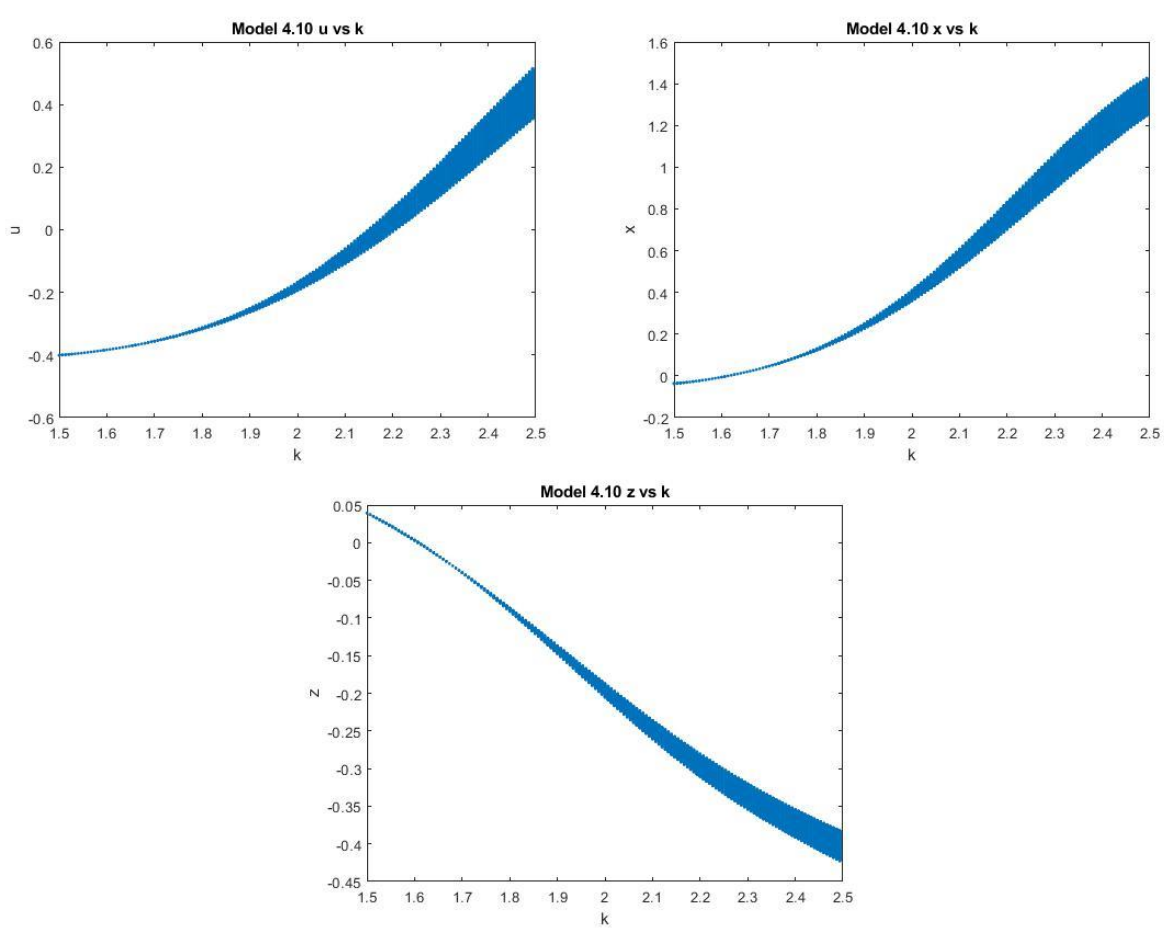

Figures 4.2.3(e). ESDDFD2 model (4.10) (i)u vs $\alpha_{5},(i i) x$ vs $\alpha_{5},(i i i) z$ vs $\alpha_{5}$, at $p=1, \alpha_{5}=0.3, k \in[1.5,2.5]$
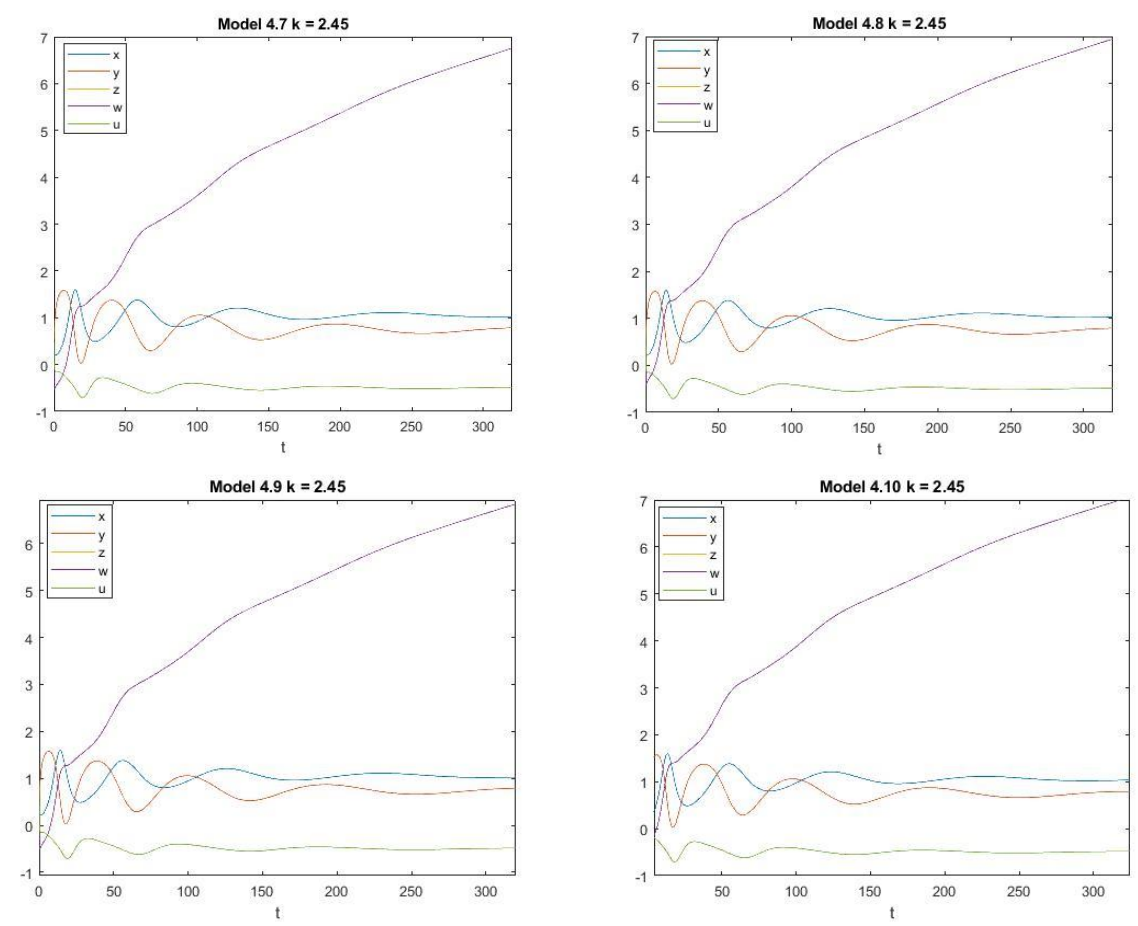

Figures 4.2.3(f). Models (4.7), (4.8), (4.9) and (4.10) profiles of $x, y, z, w$ and $u$ at $k=2.45, p=1, \alpha_{5}=0.3$

4.2.4 With fixed $\mathbf{k}=\mathbf{2}, \mathbf{p}=\mathbf{1}$ and $\boldsymbol{\alpha} \mathbf{5}=\mathbf{0 . 2 4}$. [Xin et al., 2019] concluded that system (2.4) has a hyperchaotic attractor in the $y-z-u$ and $x-y-w$ planes. 


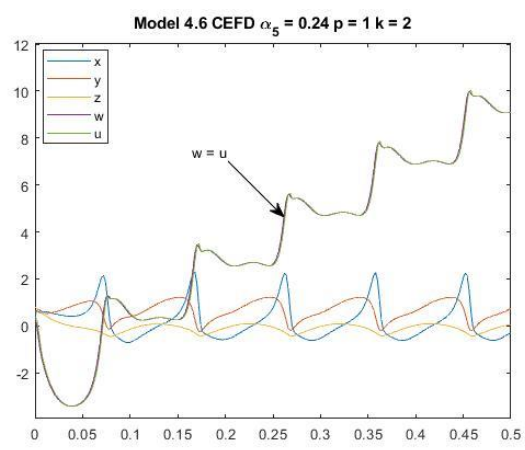

Figures 4.2.4(a). CEFD model (4.6) $k=2, p=1, \alpha_{5}=0.24$
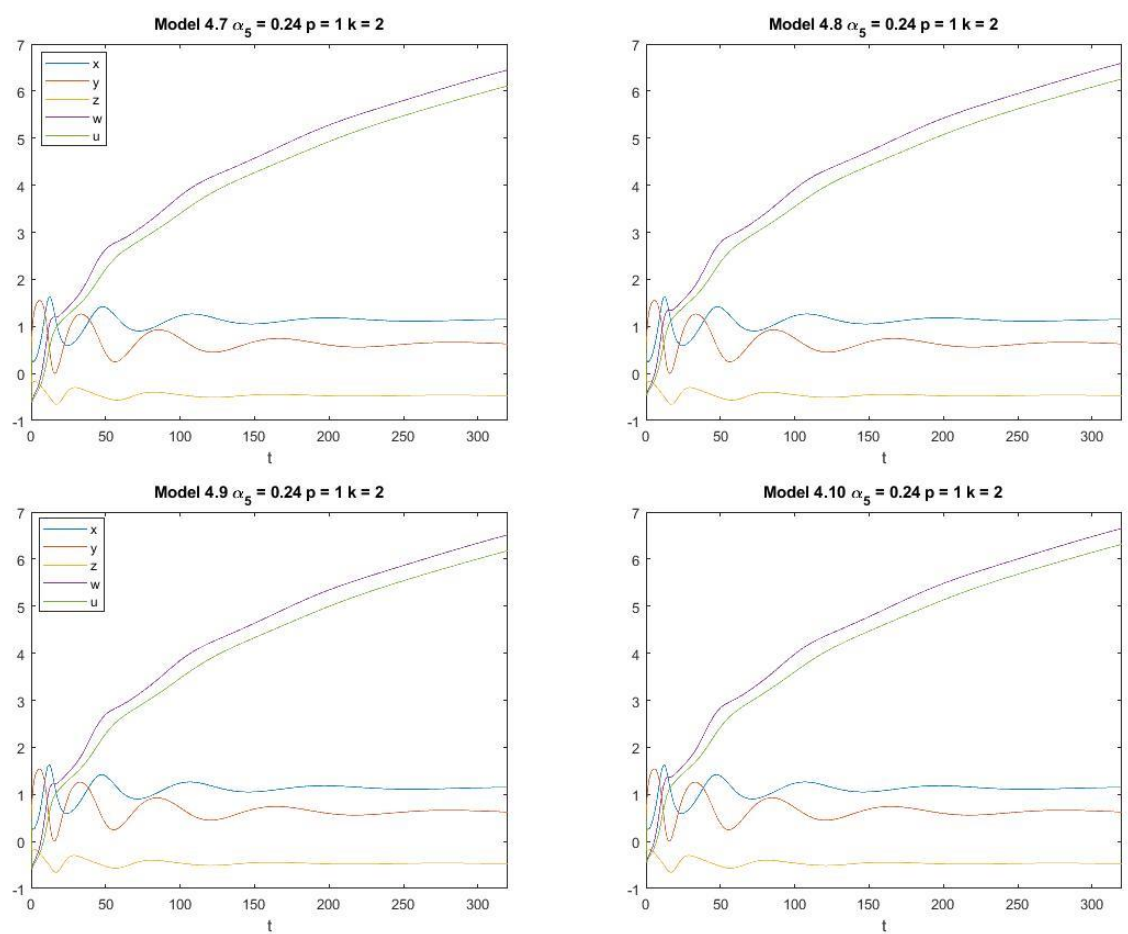

Figures 4.2.4(b). MCEFD models (4.7) through (4.10) $k=2, p=1, \alpha_{5}=0.24$

\section{Discussion}

A discrete model using the conformable Euler finite difference (CEFD) model, (2.4), was constructed in [Xin et al., 2019] and used to detect hyperchaotic behavior of the system (1.1). In this paper, a discrete model (1.2) has been constructed for the system (1.1) and the parameters from [Xin et al., 2019] used to study hyperchaos using bifurcation techniques. The discrete model (1.2) is constructed using the exact spectral derivative discretization finite difference (ESDDFD) method, a universal extension of the nonstandard finite difference method to fractional derivatives, which is designed to eliminate contrived chaos, Various cases are considered, in parallel to those considered in [Xin et al., 2019] as well as for sub-systems relevant to the construction of the discrete model (1.2) 


\section{References}

[Xin et al., 2019] Xin et al. (Baogui Xin , Wei Peng, Yekyung Kwon, and Yanqin Liu) (2019). Modeling, discretization, and hyperchaos detection of conformable derivative approach to a financial system with market confidence and ethics risk. Advances in Difference Equations (2019) 2019:138 https://doi.org/10.1186/s13662-019-2074-8

[DPC-M \& BGBC-M, 2021] D.P. Clemence-Mkhope and B.G.B. Clemence-Mkhope. The Limited Validity of the Conformable Euler Finite Difference Method and an Alternate Definition of the Conformable Fractional Derivative to Justify Modification of the Method. Mathematical and Computational Applications. 2021; 26(4):66. https://doi.org/10.3390/mca26040066

[Mick 2020] R.E. Mickens. Nonstandard Finite Difference Schemes: Methodology and Applications; World Scientific Publishing Company, Singapore, 2020

[Garba et. al] S.M. Garba, A.B. Gumel, and J.M.-S. Lubuma. Dynamically-consistent non-standard finite difference method for an epidemic model. Mathematical and Computer Modelling 53 (2011), 131-150

[Imb, 2019] Imbert, Alberto F.. Contributions to Conformable and Non-Conformable Calculus, Doctor in Mathematical Engineering Thesis, Universidad Carlos III de Madrid, 2019. https://www.researchgate.net/publication/342654962 Contributions to Conformable and non-

Conformable Calculus. (accessed May 28, 2021)

[DPC-M, 2021 arXiv(a)] D.P. Clemence-Mkhope (2021, Preprint). The Exact Spectral Derivative Discretization Finite Difference (ESDDFD) Method for Wave Models. arXiv:2106.07609 (accessed July 16, 2021)

[DPC-M, 2021arXiv(b)] D.P. Clemence-Mkhope (2021, Preprint). Spectral Non-integer Derivative Representations and the Exact Spectral Derivative Discretization Finite Difference Method for the FokkerPlanck Equation arXiv:2106.02586 (accessed July 16, 2021)

1. Liu, Y., Li, J., Wei, Z., Moroz, I.: Bifurcation analysis and integrability in the segmented disc dynamo with mechanical friction. Adv. Differ. Equ. 2018, 210 (2018)

2. Wei, Z., Moroz, I., Sprott, J.C., et al.: Hidden hyperchaos and electronic circuit application in a 5D self-exciting homopolar disc dynamo. Chaos 27(3), 033101 (2017)

3. Wei, Z., Rajagopal, K., Zhang, W., et al.: Synchronisation, electronic circuit implementation, and fractional-order analysis of 5D ordinary differential equations with hidden hyperchaotic attractors. Pramana 90(4), 50 (2018)

4. Li, C., Chen, G.: Chaos and hyperchaos in the fractional-order Rossler equations. Physica A 341, 55-61 (2004)

5. Rajagopal, K., Karthikeyan, A., Duraisamy, P.: Hyperchaotic chameleon: fractional-order FPGA implementation. Complexity 2017, 8979408 (2017)

6. El-Sayed, A., et al.: Dynamical behaviors, circuit realization, chaos control, and synchronization of a new fractional-order hyperchaotic system. Appl. Math. Model. 40(5-6), 3516-3534 (2016)

7. El-Sayed, A., Elsonbaty, A., Elsadany, A., Matouk, A.: Dynamical analysis and circuit simulation of a new fractional-order hyperchaotic system and its discretization. Int. J. Bifurc. Chaos 26(13), 1650222 (2016)

8. Mou, J., Sun, K.: Characteristic analysis of fractional-order 4D hyperchaotic memristive circuit. Math. Probl. Eng. 2017, 2313768 (2017)

9. Wang, Y., He, S., Wang, H., et al.: Bifurcations and synchronization of the fractional-order simplified Lorenz hyperchaotic system. J. Appl. Anal. Comput. 5(2), 210-219 (2015)

10. Huang, X., Zhao, Z., Wang, Z., et al.: Chaos and hyperchaos in fractional-order cellular neural networks. Neurocomputing 94(3), 13-21 (2012)

11. Huang, D., Li, H.: Theory and Method of the Nonlinear Economics. Sichuan University Press, Chengdu (1993)

12. Chen, W.: Nonlinear dynamics and chaos in a fractional-order financial system. Chaos Solitons Fractals 36, 1305-1314 (2008)

13. Wang, Z., Huang, X., Shen, H.: Control of an uncertain fractional-order economic system via adaptive sliding mode. Neurocomputing 83, 83-88 (2012)

14. Mircea, G., Neamtu, M., Bundau, O., Opris, D.: Uncertain and stochastic financial models with multiple delays. Int. J. 
Bifurc. Chaos 22, 1250131 (2012)

15. Xin, B., Chen, T., Ma, J.: Neimark-Sacker bifurcation in a discrete-time financial system. Discrete Dyn. Nat. Soc. 2010, $405639(2010)$

16. Yu, H., Cai, G., Li, Y.: Dynamic analysis and control of a new hyperchaotic finance system. Chaos Solitons Fractals 45 , 1048-1057 (2012)

17. Xin, B., Li, Y.: 0-1 test for chaos in a fractional-order financial system with investment incentive. Abstr. Appl. Anal. 2013,876298 (2013)

18. Xin, B., Zhang, J.: Finite-time stabilizing a fractional-order chaotic financial system with market confidence. Nonlinear Dyn. 79(2), 1399-1409 (2015)

19. Zhang, L., Sun, K., He, S., et al.: Solution and dynamics of a fractional-order 5-D hyperchaotic system with four wings. Eur. Phys. J. Plus 132(1), 31 (2017)

20. Wang, S., Wu, R.: Dynamic analysis of a 5D fractional-order hyperchaotic system. Int. J. Control. Autom. Syst. 15(3), 1003-1010 (2017)

21. Zheng, R., Jiang, X.: Spectral methods for the time-fractional Navier-Stokes equation. Appl. Math. Lett. 91, 194-200 (2019)

22. Xu, H., Jiang, X.: Creep constitutive models for viscoelastic materials based on fractional derivatives. Comput. Math. Appl. 73(6), 1377-1384 (2017)

23. Fan, W., Qi, H.: An efficient finite element method for the two-dimensional nonlinear time-space fractional Schrodinger equation on an irregular convex domain. Appl. Math. Lett. 86, 103-110 (2018)

24. Yang, X., Qi, H., Jiang, X.: Numerical analysis for electroosmotic flow of fractional Maxwell fluids. Appl. Math. Lett. 78, $1-8(2018)$

25. Gao, X., Chen, D., Yan, D., et al.: Dynamic evolution characteristics of a fractional-order hydropower station system. Mod. Phys. Lett. B 32(2), 1750363 (2018)

26. Wang, F., Chen, D., Zhang, X., Wu, Y.: Finite-time stability of a class of nonlinear fractional-order system with the discrete time delay. Int. J. Syst. Sci. 48, 984-993 (2017)

27. Wu, G., Baleanu, D., Huang, L.: Novel Mittag-Leffler stability of linear fractional delay difference equations with impulse. Appl. Math. Lett. 82, 71-78 (2018)

28. Wu, G., Baleanu, D., Luo, W.: Analysis of fractional non-linear diffusion behaviors based on Adomian polynomials. Therm. Sci. 21(2), 813-817 (2017)

29. Khalil, R., Al Horani, M., Yousef, A., Sababheh, M.: A new definition of fractional derivative. J. Comput. Appl. Math. 264, 65-70 (2014)

30. Abdeljawad, T.: On conformable fractional calculus. J. Comput. Appl. Math. 279, 57-66 (2015)

31. Abdeljawad, T., Al-Mdallal, Q., Jarad, F.: Fractional logistic models in the frame of fractional operators generated by conformable derivatives. Chaos Solitons Fractals 119, 94-101 (2019)

32. Acan, O., Firat, O., Keskin, Y.: Conformable variational iteration method, conformable fractional reduced differential transform method and conformable homotopy analysis method for non-linear fractional partial differential equations. Waves Random Complex Media 8, 1-19 (2018)

33. Attia, R., Lu, D., Khater, M.: Chaos and relativistic energy-momentum of the nonlinear time fractional Duffing equation. Math. Comput. Appl. 24(1), 10 (2019)

34. Bohner, M., Hatipoglu, V.: Dynamic cobweb models with conformable fractional derivatives. Nonlinear Anal. Hybrid Syst. 32, 157-167 (2019)

35. Tarasov, V.: No nonlocality. No fractional derivative. Commun. Nonlinear Sci. Numer. Simul. 62, 157-163 (2018)

36. Rosales, J., Godínez, F., Banda, V.: Analysis of the Drude model in view of the conformable derivative. Optik 178, 1010-1015 (2019)

37. Akbulut, A., Melike, K.: Auxiliary equation method for time-fractional differential equations with conformable derivative. Comput. Math. Appl. 75(3), 876-882 (2018)

38. Martinez, L., Rosales, J., Carreno, C.: Electrical circuits described by fractional conformable derivative. Int. J. Circuit Theory Appl. 46(5), 1091-1100 (2018)

39. Rezazadeh, H., Khodadad, F., Manafian, J.: New structure for exact solutions of nonlinear time fractional Sharma-Tasso-Olver equation via conformable fractional derivative. Appl. Appl. Math. 12(1), 13-21 (2017)

40. Korkmaz, A.: Explicit exact solutions to some one-dimensional conformable time fractional equations. Waves Random Complex Media 29(1), 124-137 (2019)

41. Perez, J., Gomez-Aguilar, J., Baleanu, D., Tchier, F.: Chaotic attractors with fractional conformable derivatives in the Liouville-Caputo sense and its dynamical behaviors. Entropy 20(5), 384 (2018)

42. He, S., Banerjee, S., Yan, B.: Chaos and symbol complexity in a conformable fractional-order memcapacitor system. Complexity 2018, 4140762 (2018)

43. Lu, Y., Yang, L., Liu, L.: Volatility spillovers between crude oil and agricultural commodity markets since the financial crisis. Sustainability 11, 396 (2019)

44. Erfani, G., Vasigh, B.: The impact of the global financial crisis on profitability of the banking industry: a comparative analysis. Economies 6, 66 (2018)

45. Dinoer, H., Yuksel, S., Senel, S.: Analyzing the global risks for the financial crisis after the great depression using comparative hybrid hesitant fuzzy decision-making models: policy recommendations for sustainable economic growth. Sustainability 10, 3126 (2018)

46. Li, R., Liu, W., Liu, Y., Tsai, S.B.: IPO underpricing after the 2008 financial crisis: a study of the Chinese stock markets. Sustainability 10, 2844 (2018)

47. Cavdar, S.C., Aydin, A.D.: An empirical analysis for the prediction of a financial crisis in Turkey through the use of forecast error measures. J. Risk Financial Manag. 8, 337-354 (2015)

48. Zhao, H., Zhao, H., Guo, S., Li, F., Hu, Y.: The impact of financial crisis on electricity demand: a case study of North China. Energies 9, 250 (2016)

49. Derwall, J., Koedijk, K., Ter Horst, J.: A tale of values-driven and profit-seeking social investors. J. Bank. Finance 35(8), 
2137-2147 (2011)

50. Rasmussen, D.: Adam Smith on what is wrong with economic inequality. Am. Polit. Sci. Rev. 110(2), 342-352 (2016)

51. Eslami, M., Rezazadeh, H.: The first integral method for Wu-Zhang system with conformable time-fractional derivative. Calcolo 53(3), 475-485 (2016)

52. Ilie, M., Biazar, J., Ayati, Z.: The first integral method for solving some conformable fractional differential equations. Opt. Quantum Electron. 50(2), 55 (2018)

53. Hosseini, K., Bekir, A., Ansari, R.: New exact solutions of the conformable time-fractional Cahn-Allen and Cahn-Hilliard equations using the modified Kudryashov method. Optik 132, 203-209 (2017)

54. Unal, E., Gokdogan, A.: Solution of conformable fractional ordinary differential equations via differential transform method. Optik 128, 264-273 (2017)

55. Kumar, D., Seadawy, A., Joardar, A.: Modified Kudryashov method via new exact solutions for some conformable fractional differential equations arising in mathematical biology. Chin. J. Phys. 56(1), 75-85 (2018)

56. Srivastava, H., Gunerhan, H.: Analytical and approximate solutions of fractional-order susceptible-infected-recovered epidemic model of childhood disease. Math. Methods Appl. Sci. 42(3), 935-941 (2019)

57. Kaplan, M.: Applications of two reliable methods for solving a nonlinear conformable time-fractional equation. Opt. Quantum Electron. 49(9), 312 (2017)

58. Yavuz, M., Ozdemir, N.: A different approach to the European option pricing model with new fractional operator Math. Model. Nat. Phenom. 13(1), 12 (2018)

59. Kartal, S., Gurcan, F.: Discretization of conformable fractional differential equations by a piecewise constant approximation. Int. J. Comput. Math. 25, 1-2 (2018)

60. Iyiola, O., Tasbozan, O., Kurt, A., Cenesiz, Y.: On the analytical solutions of the system of conformable time-fractional Robertson equations with 1-D diffusion. Chaos Solitons Fractals 94, 1-7 (2017)

61. Ruan, J., Sun, K., Mou, J., He, S., Zhang, L.: Fractional-order simplest memristor-based chaotic circuit with new derivative. Eur. Phys. J. Plus 133(1), 3 (2018)

62. He, S., Sun, K., Mei, X., Yan, B., Xu, S.: Numerical analysis of a fractional-order chaotic system based on conformable fractional-order derivative. Eur. Phys. J. Plus 132(1), 36 (2017)

63. Yokus, A.: Comparison of Caputo and conformable derivatives for time-fractional Korteweg-de Vries equation via the finite differencemethod. Int. J. Mod. Phys. B 32(29), 1850365 (2018)

64. Rezazadeh, H., Ziabarya, B.: Sub-equation method for the conformable fractional generalized Kuramoto-Sivashinsky equation. Comput. Res. Prog. App. Sci. Eng. 2(3), 106-109 (2016)

65. Zhong, W., Wang, L.: Basic theory of initial value problems of conformable fractional differential equations. Adv. Differ. Equ. 1, 321 (2018)

66. Tayyan, B., Sakka, A.: Lie symmetry analysis of some conformable fractional partial differential equations. Arab. J. Math. 2018, 1-12 (2018)

67. Yaslan, H.: Numerical solution of the conformable space-time fractional wave equation. Chin. J. Phys. 56(6), 2916-2925 (2018)

68. Kurt, A., Cenesiz, Y., Tasbozan, O.: On the solution of Burgers' equation with the new fractional derivative. Open Phys. $13,355-360$ (2015)

69. Khalil, R., Abu-Shaab, H.: Solution of some conformable fractional differential equations. Int. J. Pure Appl. Math. 103(4), 667-673 (2015)

70. Unal, E., Gokdogan, A., Celik, E.: Solutions of sequential conformable fractional differential equations around an ordinary point and conformable fractional Hermite differential equation (2015). Preprint. arXiv:1503.05407

71. Liu, S., Wang, H., Li, X., Li, H.: The extremal iteration solution to a coupled system of nonlinear conformable fractional differential equations. J. Nonlinear Sci. Appl. 10, 5082-5089 (2017)

72. Cenesiz, Y., Kurt, A.: The solutions of time and space conformable fractional heat equations with conformable Fourier transform. Acta Univ. Sapientiae Math. 7(2), 130-140 (2015)

73. El-Sayed, A., Salman, S.: On a discretization process of fractional-order Riccati differential equation. J. Fract. Calc. Appl. $4(2), 251-259(2013)$

74. Agarwal, R., El-Sayed, A., Salman, S.: Fractional-order Chua's system: discretization, bifurcation and chaos. Adv. Differ. Equ. 1, 320 (2013)

75. Mohammadnezhad, V., Eslami, M., Rezazadeh, H.: Stability analysis of linear conformable fractional differential equations system with time delays. Bol. Soc. Parana. Mat. 38(6), 159-171 (2020)

76. Xin, B., Chen, T., Liu, Y.: Synchronization of chaotic fractional-order WINDMI systems via linear state error feedback control. Math. Probl. Eng. 2010, 859685 (2010)

77. Yavuz, M., Ozdemir, N.: European vanilla option pricing model of fractional-order without singular kernel. Fractal Fract. 2(1), 3 (2018)

78. Baskonus, H., Mekkaoui, T., Hammouch, Z., Bulut, H.: Active control of a chaotic fractional-order economic system. Entropy 17, 5771-5783 (2015)

79. Ma, J., Ren, W.: Complexity and Hopf bifurcation analysis on a kind of fractional-order IS-LM macroeconomic system. Int. J. Bifurc. Chaos 26(11), 1650181 (2016)

80. Huang, Y., Wang, D., Zhang, J., Guo, F.: Controlling and synchronizing a fractional-order chaotic system using stability theory of a time-varying fractional-order system. PLoS ONE 13(3), e0194112 (2018)

81. Xin, B., Chen, T., Liu, Y.: Projective synchronization of chaotic fractional-order energy resources demand-supply systems via linear control. Commun. Nonlinear Sci. Numer. Simul. 16, 4479-4486 (2011)

82. Almeida, R., Malinowska, A.B., Monteiro, M.T.T.: Fractional differential equations with a Caputo derivative with respect to a kernel function and their applications. Math. Methods Appl. Sci. 41(1), 336-352 (2018)

83. Yuan, L., Yang, Q.: Parameter identification and synchronization of fractional-order chaotic systems. Commun. Nonlinear Sci. Numer. Simul. 17(1), 305-316 (2012)

84. Behinfaraz, R., Badamchizadeh, M., Ghiasi, A.R.: Parameter identification and synchronization of fractional-order chaotic systems. Appl. Math. Model. 40(7-8), 4468-4479 (2016) 
85. Belkhatir, Z., Laleg-Kirati, T.M.: Parameters and fractional differentiation orders estimation for linear continuous-time non-commensurate fractional order systems. Syst. Control Lett. 115, 26-33 (2018)

86. Pikulina, E., Renneboog, L., Tobler, P.: Overconfidence and investment: an experimental approach. J. Corp. Finance 43(4), 175-192 (2017)

87. Deaves, R., Kluger, B., Miele, J.: An exploratory experimental analysis of path-dependent investment behaviors. J. Econ. Psychol. 43(4), 175-192 (2017) 